\title{
The fate of standing variation and new mutation under climate change
}

\author{
Cheng-Yueh Lu${ }^{1}$, Cheng-Ruei Lee $e^{1,2,3 *}$
}

3

$4 \quad{ }^{1}$ Institute of Plant Biology, National Taiwan University

$5 \quad{ }^{2}$ Institute of Ecology and Evolutionary Biology, National Taiwan University

$6 \quad{ }^{3}$ Genome and Systems Biology Degree Program, National Taiwan University

7 *Correspondence to: chengrueilee@ntu.edu.tw

8 Abstract:

10 Many species face existence threat under anthropogenic climate change, and standing genetic 11 variation was proposed as a way for sessile species to adapt to novel environments. However, 12 it is still unknown whether standing genetic variants, being adaptive to current environmental 13 variability, are sufficient to guarantee future survival. Here we investigate the relative 14 importance of standing variation versus new mutations from the past to infer their future fate 15 in nature. In the wild banana species Musa itinerans where the Taiwanese populations were 16 ancient immigrants from the Chinese populations, new mutations exert larger effect size in 17 precipitation-related variables, where Taiwan contains extreme environments beyond the 18 ancestral climatic range, and new alleles have stronger association with novel environments. 19 For temperature-related variables where Taiwan is within the ancestral climatic range, standing 20 variants are more important than new mutations. The effect sizes of adaptive variants therefore 21 differ under distinct environmental pressures, supporting theoretical predictions that natural selection favors new mutations with larger effect sizes in novel environments where the population is far from the adaptive peak. Despite their importance, large-effect variants also have higher mismatch and may be more vulnerable to future environmental perturbation, leaving minor-effect variants the main source of adaptive response to rapid anthropogenic climate change. Our work provides a support in natural environment to the previous conclusions from theoretical modeling and microbial experiments in well-controlled lab conditions. 


\section{Introduction}

Anthropogenic climatic change posts an imminent threat to most organisms. For large and sessile plant species with long generation time, the speed of migration may not keep up with environmental change, and therefore phenotypic plasticity and genetic variation in the population may allow their survival under novel environments [1,2]. Adaptive genetic variation originates from standing variants before or new mutations after environmental change. Since anthropogenic climate change greatly outpaces natural mutation, the amount of standing genetic variation is therefore critical for the rapid response of a population to changing environments [3]. It remains unclear, however, whether standing variation is sufficient to guarantee future survival, given that they were mostly adaptive to the present range of climatic variability. While it may be difficult to perform manipulative experiments in the field to compare the effects of new mutations (NM) and standing variation (SV), one could investigate NM and SV during climatic change in the past.

Adaptation could happen through genetic variants that differ in their origins (NM or SV) or effect sizes (Mendelian genes with major effects or polygenic variants with minor effects). However, how these factors interact and respond to environmental pressures remains relatively uninvestigated. For example, does SV and NM differ in their relative number or effect sizes towards environmental adaptation, and how does this relationship change with different types of environmental factor? For adaptive new mutations that were fixed when facing environment change, Fisher first predicted primarily small allelic effects [4] while Kimura emphasized intermediate effects [5]. Orr, later considering the entire adaptive walk, concluded the evolution towards a novel adaptive peak should first happen through fixation of large-effect mutations and later by small-effect polymorphisms [6]. While this was supported by some studies, the majority of these are microbial experimental evolution in well-controlled environments $[7,8]$, and few have specifically compared the effects of NM and SV. To test whether this idea holds in nature, empirical investigations on natural populations are needed.

Taiwan is well-suited for such studies: Unlike oceanic islands such as Hawaii, Taiwan is a continental island where most species originated from the East Asian continent with recurrent gene flow [9]. The land bridge between Taiwan and China during the glacial maximum allowed exchange of SV, and the isolation during interglacial periods enabled the development of NM. Here we investigate the genomic basis of environmental adaptation of a wild banana species, Musa itinerans, whose habitats in Taiwan are considered peripheral from ancestral area reconstructions [10], providing an opportunity to distinguish SV from NM, as well as their response to ancestral versus novel adaptive landscapes. We investigated how past events (SV versus NM) influence present adaptation and whether local adaptation may persist under future anthropogenic climate change. 


\section{Results}

\section{Environmental adaptation in Musa itinerans}

We first sampled Musa itinerans at 24 populations across Taiwan (Fig. 1a; fig. S1a; table S1) and investigated the population structure using 14 microsatellites (table S2). Environmental Niche Modeling (with 483 occurrence points from field survey and Google Street View) reported species distribution (Fig. 1b) in line with the previous statement that Musa itinerans inhabits sunny valleys, watersheds, and hillsides with gentle slopes [11]. Populations differentiated mostly between east and west (fig. S1b). The most unsuitable environments lay within the Central Mountain Range and the southwestern plains (Fig. 1b), respectively corresponding to low annual mean temperature (BIO1) and low precipitation of driest quarter (BIO17), the two most important bioclimatic variables determining species distribution (MaxEnt permutation importance [12]: 36.7 for BIO1 and 27.3 for BIO17).

To test for local adaptation, we examined the pattern of "isolation by adaptation" [13], a process where differential local adaptation restricted effective gene flow and promoted genetic differentiation among populations, by dissecting geographic and environmental effects on genetic differentiation. The strait-line "fly-over" geographical distance, calculated as the straight-line distance between locations, does not explain patterns of genetic differentiation (Mantel's $r=0.146$ and $P=0.062$ ). However, if we considered that Central Mountain Range lacks corridors for M. itinerans to disperse (Fig. 1b), this fly-over geographical distance could be too unrealistic. We therefore used resistance distance, calculated from the route with least resistance among populations on the niche suitability map (Fig. 1b), to represent the "realized" geographical distance (Fig. 1c) and found that genetic differentiation was significantly associated with resistance (Mantel's $r=0.226$ and $P=0.006$ ). The environmental Mahalanobis distance of bioclimatic variables also showed strong association with genetic differentiation (Mantel's $r=0.298$ and $P=0.005$ ). Given that the environmental distance could be strongly dependent on geography, we performed Partial Mantel test to control the geographical effect. After controlling for realized geographic distance (resistance distance), genetic differentiation still correlates with the Mahalanobis environmental distance (Mantel's $r=0.250$ and $P=0.012$ ), suggesting differential local adaptation is associated with genetic variation.

\section{Standing variation versus new mutations}

100 To identify genomic regions associated with environmental adaptation, we performed pooled sequencing for each population. SNPs were separated into standing variation (SV: both alleles exist in Taiwan and China) or new mutations (NM, polymorphic only in Taiwan). SV outnumbered NM in both adaptive (identified with Bayenv [14]) and non-adaptive SNPs, and after controlling for the overall number of SNPs in SV and NM, SV were further enriched among adaptive polymorphisms (table S3). However, since adaptive SNPs also have higher minor allele frequency (MAF) (fig. S2a), this pattern could be confounded: SV are more likely 
to have higher MAF than NM, and SNPs with higher MAF may be more likely detected as adaptive due to higher statistical power. We therefore performed the same test with a subset where the adaptive and non-adaptive SNPs have similar allele frequencies (ranging from the first quantile of adaptive MAF to the third quantile of non-adaptive MAF separately for each bioclimatic variable; fig. S2a). In this case as well, SV are still disproportionately abundant

112 (table S4), suggesting SV are more likely than NM to become environment-associated SNPs.

113 Another potential confounding factor is the geographic extent of variants: if most NM resulted

114 from mutations restricted to a few local populations, the limited distribution prevents

115 environment association for NM. We therefore compared the number of Taiwanese populations

116 containing the minor alleles for SV and NM, respectively. Contrary to the direction predicted

117 by the aforementioned confounding factor, the geographic extent of minor alleles for SV is

118 slightly smaller than NM (15.8 populations for SV and 16.3 for NM, $P<0.001)$.

119 In addition to SNP number, do NM and SV differ in their directions of effect? Under the null hypothesis that (I) the effects of NM are equally likely to facilitate adaptation to the ancestral or novel environments and (II) natural selection is equally likely to fix NM facilitating adaptation towards either direction, we expected no enrichment of new alleles in either environment. When we separated the Taiwanese populations into those within the Chinese ancestral environmental range and those with novel environments, frequencies of putatively adaptive new alleles in NM SNPs were higher in the latter set of populations, with precipitation of driest quarter (BIO17) and precipitation of coldest quarter (BIO19) showing the strongest effect (Fig. 2a; fig. S2b). Given that the directions of mutation effects should be random, these results suggest that new variants facilitating adaptation to novel environments are more likely to be retained by selection.

The results above suggest SV might be more important than NM in terms of enriched number of variants with environment association, and adaptive NM, while lower in number, are more associated with novel environments. On the other hand, the number of candidate SNPs does not necessarily reflect the overall importance of SV over NM, since the effect size also needs to be considered. To investigate the effect size of SV and NM in environmental adaptation, we compared their Bayes factors from Bayenv and focused on two bioclimatic variables that were important in determining species distribution, annual mean temperature (BIO1) and precipitation of driest quarter (BIO17). The two variables exhibit opposite patterns (Fig. 2c): While in BIO17 and other related precipitation variables (fig. S3a) NM consistently had higher Bayes factor and therefore stronger effect size than SV, in BIO1 and other related temperature variables (fig. S3a) we observed the reverse. The same trend was observed when we estimated the effect size with gradient forest $[15,16]$ (Fig. 2b and 2c; fig. S3b and S3c): BIO17 was the most important factor for differential local adaptation, and NM had stronger effects than SV. On the other hand, BIO1 was the least important factor where SV had stronger 
the amount of allele frequency variation explained by environmental gradients. Assuming a simple linear relationship between allele frequency and environment, the value of $r^{2}$ only represents how well each data point (a population) fits along the regression line. We were, however, also interested in the regression slope: the amount of allele frequency changes along environmental gradients (fig. S3d). Again, BIO17 had the largest overall slope among all bioclimatic variables (fig. S3e), with NM being significantly higher than SV (Fig. 2c). BIO1 had the lowest overall slope, again with the reversed pattern. Given that the MAF of adaptive NM and SV SNPs are similar, there is no need to control for allele frequency in these tests (fig. $\mathrm{S} 4 \mathrm{a}$ ). Therefore, NM with larger effect size per SNP (as estimated by Bayenv Bayes factor, gradient forest $r^{2}$ weighted importance, and gradient forest slope) were associated with the adaptation to novel environments outside of the ancestral niche space, consistent with previous population genetics modeling results [4-6].

The observed patterns could be integrated with the unique climate of Taiwan. In comparison to the rest of the species range, northern Taiwan experiences northeastern monsoons during winter and has higher precipitation during the typical winter dry season (Fig. 3b). This pattern has been maintained since at least the last glacial maximum (Fig. 3d). The novel environments might impose novel adaptive optima to the immigrant population from China. The response to selection imposed by these environmental gradients is strong (with highest Bayes factor, $r^{2}$, and slope among all bioclimatic variables; fig. S3a, S3c, and S3e) especially for NM, where new alleles are strongly associated with novel environments (fig. $\mathrm{S} 2 \mathrm{~b}$ ). More importantly, for this major driver of adaptation (BIO17), the greatest increment of gradient forest importance lies between $200 \mathrm{~mm}$ and $300 \mathrm{~mm}$ (Fig. 2b), a range also distinguishing the novel Taiwanese versus ancestral Chinese environments (Fig. 3b). This suggests that the majority of differential local adaptation is associated with such novel-versusancestral environmental differences. Annual mean temperature (BIO1) is the other extreme: the environmental gradient within Taiwan is well within the ancestral Chinese environmental range (Fig. 3a), which can also be traced back to the last glacial maximum (Fig. 3c). It is likely that SV already contained genetic variants adaptive to such environmental gradients and are therefore more important than NM (Fig. 2c). In summary, we observed adaptation happening through the assortment of SV for a new territory with similar adaptive landscape and optimum (BIO1; Fig. 2c, 3a, and 3c). For adaptation to novel environments and a new adaptive landscape (BIO17; Fig. 2c, 3b, and 3d), NM with larger effect sizes were more likely favored by natural selection. Our results are therefore consistent with Orr's model ${ }^{3}$, providing one of the few examples in nature.

One key point of this study is the correct designation of SV or NM. It is possible that some SV SNPs were mis-assigned as NM if we missed an allele in China, most likely for SNPs with low MAF. We addressed this issue with the following: (I) The Taiwanese populations were nested within China in the phylogenetic tree (fig. S4b) and contained much less genetic 
variation (fig. S4c). Due to the stronger genetic drift in Taiwan than China, it is less likely that an originally SV SNP would retain both alleles in Taiwan but lose one in China. (II) In the extreme case, assuming 50\% of NM SNPs were mis-assigned from SV, we performed 100 new analyses, each randomly assigning $50 \%$ of NM SNPs back to SV. These new analyses yielded similar results, with NM having higher effect sizes than SV in precipitation-related variables (fig. S5a). (III) Since MAF are correlated between Taiwan and China (Spearman's rank correlation $\rho=0.35, P<0.001$ ), we performed analyses with top 50\% MAF SNPs in Taiwan, thereby reducing the chance of missing minor alleles in China. The results are qualitatively the same (fig. S5b-d).

\section{Fate of adaptive variants under future climate change}

In addition to understanding how past events (SV versus NM) affected present adaptation, we are also concerned with how these factors affect the future of this species under anthropogenic climate change. We used Bayenv to investigate the fate of currently adaptive SNPs under 16 different future climatic scenarios. Currently adaptive SNPs retaining high association with future environments are classified as "retention", while those no longer associated with future environments are "disruption". Different from the present pattern of SV being enriched in adaptive SNPs, we saw no clear tendency for SV or NM enriched towards retention or disruption (table S5), suggesting both types of genetic variants will be affected by climate change regardless of their previous origin.

We further used the genetic offset values from gradient forest to estimate genetic mismatch for SV and NM separately, which is associated with the magnitude of allele frequency turnover perturbed by future climatic conditions [16]. After projecting the genetic mismatch of SV and NM on the map of Taiwan for all 16 predicted future scenarios, we found SV generally has higher mean (paired t-test, $t=3.296, P=0.005$, Fig. 4A) while NM has higher maximum genetic offset across Taiwan (paired t-test, $t=-8.608, P<0.001$, Fig. 4B). We further averaged the genetic offset map across all 16 future scenarios (Fig. 4C-E). There are more geographical grids with SV offset higher than NM (12,711 square kilometers) and less grids with NM offset higher than SV (8,707 square kilometers), consistent with the higher mean genetic offset for SV than NM. On the other hand, the most extreme genetic offset values were often observed for NM rather than SV (Fig. 4D-E).

Geographic regions where NM has much higher genetic offset (maladaptation towards anthropogenic climate change) than SV include northwestern mountainous and eastern coastal regions. Interestingly, these regions appear to have BIO17 values (precipitation of driest quarter) close to 200, where NM has much sharper transition of adaptive allele frequency than SV (Fig. 2B and 3B). To further examine such association, we cut the Taiwan map into 20 bins of equal grid numbers based on their current BIO17 value (excluding grids with species-wide Maxent suitability $<0.2$ ) and estimated the mean genetic offset values of grids within each bin. 
221 Indeed, NM has much higher genetic offset in regions with BIO17 value near 200 (Fig. 4F).

222 Such pattern exists not only for BIO17 but also for many other bioclimatic variables (fig. S6)

223 where genetic variants (either SV or NM) tend to have higher genetic offset in environments

224 with sharp increase in gradient forest's cumulative importance (high delta importance per unit

225 environmental gradient in fig. S6), indicating populations closer to the threshold of sharp

226 adaptive allele frequency change might be more vulnerable to climate perturbation.

Taken together, for adaptation in the past, NM is relatively less enriched in number but has higher effect size per SNP. For maladaptation towards the future, here we show a similar pattern: NM has weaker genetic offset than SV in the majority of geographic regions, but NM tends to have the overall highest genetic offset values in locations near the sharp transition of gradient forest's cumulative importance. In other words, while NM has higher effect size and sharper allele frequency transition for adaptation in the past to novel environments outside of the ancestral range, such property may also make NM more vulnerable to climate change in the future, especially near the transition zone.

\section{Discussion}

Assessing the within-species variation in climate association is the crucial first step to understand species susceptibility to fluctuating environment, and the relative importance of standing variation (SV) and new mutations (NM) in adaptation has long been debated [17-22]. In this study, we investigate how past genetic variation (SV and NM) contribute to the adaptation of present environments as well as how these factors together affect the future fate of a species under anthropogenic climate change.

For a population facing environmental change and therefore a novel adaptive landscape, previous population genetics models have documented the effect size distribution of new mutations fixed by natural selection. Considering the entire adaptive walk of a population facing novel environments, Orr's model predicted the distribution of effect sizes where early substitutions have larger effect than later ones [6]. Consistent with Orr's prediction, we show that NM have stronger effect size than SV in precipitation-related variables, where Taiwan exceeds the ancestral climatic range in China (and therefore the migrating population was far away from the optimum in the new adaptive landscape). This pattern is reversed for temperature-related variables, where Taiwan has similar environmental range as China. Here we provide another perspective to recent research showing that SV contributes to adaptation $[21,22]$. We show that SV indeed dominate over NM in number [21]. However, the effect size of SV and NM hinges on environmental conditions: natural selection may prefer new mutations contributing to the adaptation to the new rather than the old environment, and the effect sizes of NM tend to be higher than SV under such conditions. Looking towards the future, our results imply that standing genetic variation in a population may not be sufficient for the adaptation to 
rapid anthropogenic climate change.

Projecting into the future, we observed similar patterns. Both SV and NM could be affected by anthropogenic climate change depending on their genetic architecture of climatic adaptation: SV has slightly larger genetic offset (maladaptation to anthropogenic climate change) than NM in the majority of geographic regions, but the largest genetic offset values were mostly observed for NM SNPs in locations near the sharp transition of gradient forest's cumulative importance. On one hand, the higher effect sizes of NM SNPs suggest their importance in past adaptation towards novel environments. On the other, the high effect size is accompanied by sharp allele frequency transitions across environmental gradients, making NM SNPs highly sensitive (or even more vulnerable) to environmental perturbations in the future. Our results also suggest that, under frequent environmental changes such as repeated glacial cycles, although natural selection might favor new mutations with higher effect sizes under novel environments, such genetic variants might more likely be mal-adaptive during the next round of environmental change due to their high effect sizes. In other words, adaptive variants of high effect sizes may be either strongly favored or disfavored by different rounds of environmental changes, making these high-effect variants less likely to be retained as the standing variation for future. This is consistent with previous notions that genetic variants with higher effect sizes might be more likely to "overshoot" the adaptive peak [4] and may partially contribute to the observations that most adaptations have polygenic architecture [23]. The results also echo a recent experimental evolution study showing non-synonymous mutations are more difficult to be retained in antagonistic changing environments [24]. For the future fate of organisms living in natural environments, further large-effect novel mutations facilitating the adaptation to new environments may happen in the future, although they will be strongly outpaced by anthropogenic climate change.

Methods:

\section{Sample collection and DNA extraction.}

Field work was conducted during 2017 (August - December) and 2018 (January - May). We sampled Musa itinerans at 24 sites across Taiwan (fig. S1a; table S1). Fresh leaves were harvested from nine to fifteen individuals at each site. Total genomic DNA was extracted using the standard CTAB extraction method [25]. Since other commercial Musa species were also grown in Taiwan, we developed an indel marker for species delimitation. From previous studies [26-29], we identified a 6-bp insertion specific for the Taiwanese M. itinerans in the atpB-rbcL region of chloroplast. We designed a primer pair (5'-GAAGGGGTAGGATTGATTCTCA-3';

295 5'-CGACTTGGCATGGCACTATT-3') and used amplicon size to confirm all collected samples are Taiwanese M. itinerans. 


\section{Simple sequence repeat genotyping and analysis.}

SSR primer sequences used in this study were originally developed for the genus Musa [30,31], which were then applied on Musa itinerans. Previously documented primer sequences were first searched against the Musa acuminata DH-Pahang genome version 2 [32] on Banana Genome Hub (https://banana-genome-hub.southgreen.fr/) to check specificity as having only one amplicon, resulting in 26 primer pairs. These primers were then experimented to check specificity on M. itinerans, resulting in 14 pairs (table S2). We modified each pair of primers by capping the 5' end of forward primers with M13 sequences (CACGACGTTGTAAAACGAC) and inflorescent molecules [33]. SSR amplicons were run through capillary electrophoresis and the length of each allele was recorded.

Population structure of 20 populations (table S1) was analyzed with 14 SSR markers (table S2). Lowland populations (C35H, WFL, THNL, PTWT, P199H, MLLYT, HDPG, TTL, NAJY, HLCN, NXIR, and DFR), east transect populations (TPS300, TPS500, TPS700, and TPS900), and west transect populations (XT400, XT700, XT1200, and XT1500) were used in the analysis. We inferred the ancestry of 244 individuals with STRUCTURE 2.3.4 [34,35], parameterizing a run to have (I) run length of burnin and after-burnin period of 100,000, (II) admixture ancestry model, and (III) independent allele frequency model, further setting 20 runs for each $K$ value.

To investigate the association among genetic, geographical, and environmental distance, we generated these distance matrices. Genetic distance was calculated by GenAlEx 6.503 [36,37] from 14 SSR markers; straight geographical distance (the fly-over distance) was generated by ArcGIS 10.5 (http://desktop.arcgis.com/en/); environmental distance was measured as Mahalanobis distance to address the correlation among nine bioclimatic variables (below). In addition to the fly-over geographical distance which assumes organism dispersal ignores landscapes, we further calculated as resistance distance the cumulative cost along the least cost path (below). Matrix association was examined under Mantel and Partial Mantel tests. Statistical significance was examined with 1,000 permutations. We performed Mantel tests on (I) genetic distance $v s$. fly-over distance, (II) genetic distance $v$ s. resistance distance, (III) genetic distance vs. Mahalanobis environmental distance, and Partial Mantel tests on (IV) genetic distance vs. Mahalanobis environmental distance while controlling for resistance distance.

\section{Species distribution modeling.}

Current and future species distribution models were built for Musa itinerans using presence-only data (483 occurrence points) obtained from field survey and Google Street view. Occurrence points were then reduced to 204 cells by the removal of co-occurring presence data within the same $1 \times 1 \mathrm{~km}$ grid. MaxEnt 3.4.1 [12], implemented with the maximum entropy 
modeling approach, reports an overall niche suitability and the importance of predictors by analyzing the presence-only data as well as background (psuedo-absence) data distribution [3840]. We downloaded from WorldClim database version 1.4 (http://worldclim.org/) spatial layers of 19 present-day bioclimatic variables based on high-resolution monthly temperature and rainfall data [41]. Layers were selected at spatial resolution of 30 arc-second and with a mask that ranges $119.25-122.47^{\circ} \mathrm{E}$ and $21.76-25.49^{\circ} \mathrm{N}$ covering Taiwan. Variables showing high dependence (Pearson's correlation coefficient $>0.9$ calculated from ENMTools [42]) from each other were removed, resulting in nine final variables: $\mathrm{BIO} 1-$ mean annual temperature, $\mathrm{BIO} 2$ - mean diurnal range, $\mathrm{BIO} 3$ - isothermality, $\mathrm{BIO} 7$ - temperature annual range, $\mathrm{BIO} 12$ - annual precipitation, $\mathrm{BIO} 15$ - precipitation seasonality, $\mathrm{BIO} 16$ - precipitation of wettest quarter, BIO17 - precipitation of driest quarter, and BIO19-precipitation of coldest quarter (table S1).

Present species distribution model was constructed using the default optimization settings in MaxEnt, except the regularization set to three. We tested the predictive model by ten-fold cross-validation which was carried out by randomly partitioning the data into ten equally sized subsets and then replicating models while omitting one subset in turn. In each turn, the predictive model was built using nine subsets as training data and evaluated using the other subset as test data. The output of the predictive model is the probability of presence, or called suitability, and we averaged the ten runs to have an averaged suitability.

To predict the species distribution under different scenarios of future climate change, we projected the present-day model onto eight future climatic conditions combining two periods (2050 and 2070) and four Representative Concentration Pathways (RCP 2.6, RCP 4.5, RCP 6.0, and RCP 8.5). Future climatic layers were obtained from the WorldClim database at spatial resolution of 30 arc-second and were developed based on two general circulation models: the Community Climate System Model [43], CCSM, and the Model for Interdisciplinary Research on Climate [44], MIROC. Species distribution models for the future were carried out using the same settings described above.

To estimate the least cost path between populations, we first generated the resistance surface by taking the reciprocal of suitability. Resistance and suitability is simply a monotonic transformation in which locations with higher suitability exhibit lower resistance. Pairwise least cost path was then measured among 20 populations from the resistance surface, performed by SDM Toolbox v2.3 [45]. While least cost path is the single line with least overall cost, we also constructed the least cost corridor between populations, allowing $1 \%, 2 \%$, or $5 \%$ higher cost than the least cost value. In essence, the least cost corridors represent the realized dispersal routes of organisms along suitable habitats.

\section{Sequencing library construction and SNP identification.}

We conducted whole genome pooled-sequencing [46] for each population (table S1), 
resulting in 24 pooled-sequencing libraries. Equal amount of DNA from ten individuals at each population were pooled, except for the PTWT population where only nine individuals were available. A library with 300-400 bp insert size for each pool was prepared using NEBNext Ultra II DNA Library Prep Kit (New England Biolabs). Libraries were then sequenced with 150 bp paired-end on the HiSeq X Ten platform.

Illumina reads were then trimmed with SolexaQA [47], followed by the removal of adaptor sequences with cutadapt [48], subsequently mapped to the Musa itinerans reference genome assembly ASM164941v1 [49] with BWA 0.7.15 [50]. Picard Tools (http://broadinstitute.github.io/picard) was used to mark duplicated read pairs, and the genotypes were called following GATK 3.7 best practice [51].

For the 24 pooled samples, we filtered out sites with more than two alleles, indels, quality $(\mathrm{QUAL})<30$, quality by depth $(\mathrm{QD})<2$, call rate $<0.74$, and depth $(\mathrm{DP})>$ genome-wide average depth plus three standard deviations, resulting in 4,200,177 SNPs. SNPs with minor allele frequency $(\mathrm{MAF})<0.05$, missing data in any of the pooled-seq sample, and DP per sample $<20$ were further filtered out, resulting in 1,256,894 SNPs.

To investigate the relationship between Taiwanese and Chinese M. itinerans, we downloaded public data from 24 Chinese accessions (SRR6382516 - SRR6382539) [52]. SNPs were called using all 24 Chinese and the 24 Taiwanese samples together following the pipeline described above. We did not perform any site filtering for this joint data set since the main objective is to investigate whether specific SNPs in Taiwan also existed in China as SV. This dataset has 18,442,853 SNPs. SRR6382532 was excluded due to high missing rate. Only when evaluating the averaged expected heterozygosity between Taiwanese and Chinese populations did we filter out sites with indels and QUAL $<30$, resulting in 15,591,923 SNPs.

To assess the phylogeny of our Taiwanese populations and Chinese accessions, we downloaded Musa acuminata sequence (SRR7013754) as an outgroup. SNPs were called using one $M$. acuminata species, 24 Chinese and 24 Taiwanese samples together following the pipeline described above. We filtered out sites with more than two alleles, indels, QUAL $<30$, and call rate $<0.9$, resulting in 12,693,687 SNPs. This dataset also excluded SRR6382532.

\section{Environmentally-associated SNP identification.}

We used Bayenv 2.0 [14] to search for SNPs highly associated with environmental variables. Bayenv estimates the relationship between SNPs and environments while controlling the whole-genome population structure from a subset of loose linkage-disequilibrium SNPs.

406 Loose linkage-disequilibrium SNPs were formed by sampling one SNP from scaffolds more 407 than $10 \mathrm{~kb}$ and less than $100 \mathrm{~kb}$, two SNPs from scaffolds more than $100 \mathrm{~kb}$ and less than 500 $408 \mathrm{~kb}$, three SNPs from scaffolds more than $500 \mathrm{~kb}$ and less than $1000 \mathrm{~kb}$, and four SNPs from scaffolds more than $1000 \mathrm{~kb}$. We then, for each bioclimatic variable, defined as the adaptive 
410 SNPs ones exhibiting top 1\% Bayes factor and top 5\% rho value (a nonparametric correlation 411 coefficient capable to reduce outlier effects).

412 We further investigated the fate of currently adaptive SNPs under anthropogenic climate 413 change, performing the same Bayenv analyses of currently adaptive SNPs using future climatic

414 conditions. We included two time periods (2050 and 2070) and four Representative 415 Concentration Pathways (RCP 2.6, RCP 4.5, RCP 6.0, and RCP 8.5) from two general 416 circulation models, CCSM [43] and MIROC [44], resulting in 16 future climatic conditions. If 417 a currently adaptive SNP remains strongly associated with environments, it should exhibit 418 Bayes factor above the current threshold. We then defined as "retention" a currently adaptive 419 SNP constantly exhibiting Bayes factor above the current adaptive threshold in all future 420 scenarios, and defined as "disruption" a currently adaptive SNP exhibiting Bayes factor above the current adaptive threshold in none of the future scenarios.

422

\section{The gradient forest method and genetic offset.}

We used a novel method, gradient forest $[15,16]$, to estimate the effect of environmental gradients on allele frequency differences among populations. Gradient forest is a regressiontree based machine-learning algorithm using environmental variables to partition SNP allele frequencies. The analysis was done separately for each SNP. The resulting "importance" measures how much of the variation in allele frequency was explained by partitioning the populations based on a specific value in an environmental variable. By making multiple regression trees (thus generating a random forest) for a SNP, the goodness-of-fit $r^{2}$ of a random forest is measured as the proportion of variance explained by this random forest, which is then partitioned among environmental variables in proportion to their conditional importance. Such SNP-wise importance of each environmental variable is then averaged across SNPs belonging to the standing variation (SV) or new mutations (NM) group, resulting in the overall importance (of each environmental variable). In the end, one could obtain the relation curve between environmental gradient and cumulative importance (analogous to the cumulative $r^{2}$, proportion of allele frequency differences among populations explained by environments). This curve has two properties. First, the highest point of the cumulative importance curve denotes the overall association between a climatic variable and allele frequency, and we used this to represent the effect size of these SNPs. Second, when traversing along the environmental gradient, a sudden increase of cumulative importance at some environmental value (for example, $20^{\circ} \mathrm{C}$ ) denotes populations on either side of this environmental cutoff have very different allele frequency compositions. In other words, this represents a threshold factor for local adaptation.

One can use this cumulative importance curve to estimate the effect of future environmental change on local populations. In the example above, a population's local temperature increased from $19^{\circ} \mathrm{C}$ to $21^{\circ} \mathrm{C}$ due to climate change would require larger allele frequency shift than another population whose local temperature changed from $17^{\circ} \mathrm{C}$ to $19^{\circ} \mathrm{C}$. 
448 The "genetic offset" could then be calculated as the Euclidean distance between cumulative

449 importance corresponding to the contemporary environmental value and that corresponding to

450 the future environmental value, considering all bioclimatic variables together. Genetic offset

451 can then be considered to be the magnitude of genetic change needed for a population to be

452 still adaptive in the face of climate change.

453

\section{Regression slope.}

455 The regression slope is not given by gradient forest, since it only reports the $r^{2}$ importance 456 estimate. Thus, we introduced the simple linear regression $y=\alpha+\beta x$ to measure the regression 457 slope. We took $y$ as the allele frequency, $x$ as the standardized bioclimatic variable, and $\beta$ 458 (slope) as the measurement of the amount of allele frequency changes along environmental 459 gradients. By fitting simple linear regression with the general least-square approach, $\beta$ can

460 then be expanded to $r_{x y} \frac{s_{y}}{s_{x}}$, where $r_{x y}$ is the correlation coefficient (the square root of gradient

461 forest measured "importance") between $x$ (environment value) and $y$ (allele frequency), and $462 s_{x}$ and $s_{y}$ are the standard deviation of $x$ and $y$. 
References:

1. Ghalambor CK, McKay JK, Carroll SP, Reznick DN (2007) Adaptive Versus NonAdaptive Phenotypic Plasticity and the Potential for Contemporary Adaptation in New Environments. Functional Ecology 21: 394-407.

2. Jump AS, Peñuelas J (2005) Running to stand still: adaptation and the response of plants to rapid climate change. Ecology Letters 8: 1010-1020.

3. Barrett RDH, Schluter D (2008) Adaptation from standing genetic variation. Trends Ecol Evol 23: 38-44.

4. Fisher RA (1930) The genetical theory of natural selection. Oxford, England: Clarendon Press. xiv, 272-xiv, 272 p.

5. Kimura M (1983) The Neutral Theory of Molecular Evolution. Cambridge: Cambridge University Press.

6. Orr HA (1998) The population genetics of adaptation: The distribution of factors fixed during adaptive evolution. Evolution 52: 935-949.

7. Barrick JE, Yu DS, Yoon SH, Jeong H, Oh TK, et al. (2009) Genome evolution and adaptation in a long-term experiment with Escherichia coli. Nature 461: 1243-1247.

8. Gifford DR, Schoustra SE, Kassen R (2011) The Length of Adaptive Walks Is Insensitive to Starting Fitness in Aspergillus Nidulans. Evolution 65: 3070-3078.

9. Bramwell D, Caujapé-Castells J (2011) The Biology of Island Floras. Cambridge: Cambridge University Press.

10. Janssens SB, Vandelook F, De Langhe E, Verstraete B, Smets E, et al. (2016) Evolutionary dynamics and biogeography of Musaceae reveal a correlation between the diversification of the banana family and the geological and climatic history of Southeast Asia. New Phytol 210: 1453-1465.

11. Chiu HL (2004) The collection, evaluation and analysis of genetic diversity of Musa formosana (Warb.) Hayata native in Taiwan [thesis]: National Taiwan University. 1$180 \mathrm{p}$.

12. Phillips SJ, Anderson RP, Schapire RE (2006) Maximum entropy modeling of species geographic distributions. Ecological Modelling 190: 231-259.

13. Nosil P, Egan SP, Funk DJ (2008) HETEROGENEOUS GENOMIC DIFFERENTIATION BETWEEN WALKING-STICK ECOTYPES: “ISOLATION BY ADAPTATION" AND MULTIPLE ROLES FOR DIVERGENT SELECTION. Evolution 62: 316-336.

14. Gunther T, Coop G (2013) Robust identification of local adaptation from allele frequencies. Genetics 195: 205-220.

15. Ellis N, Smith SJ, Pitcher CR (2012) Gradient forests: calculating importance gradients on physical predictors. Ecology 93: 156-168.

16. Fitzpatrick MC, Keller SR (2015) Ecological genomics meets community-level modelling 
of biodiversity: mapping the genomic landscape of current and future environmental adaptation. Ecol Lett 18: 1-16.

17. Barton NH (2000) Genetic hitchhiking. Philos Trans R Soc Lond B Biol Sci 355: 15531562.

18. Linnen CR, Kingsley EP, Jensen JD, Hoekstra HE (2009) On the origin and spread of an adaptive allele in deer mice. Science 325: 1095-1098.

19. Jones FC, Grabherr MG, Chan YF, Russell P, Mauceli E, et al. (2012) The genomic basis of adaptive evolution in threespine sticklebacks. Nature 484: 55-61.

20. Reid NM, Proestou DA, Clark BW, Warren WC, Colbourne JK, et al. (2016) The genomic landscape of rapid repeated evolutionary adaptation to toxic pollution in wild fish. Science 354: 1305-1308.

21. Lai YT, Yeung CKL, Omland KE, Pang EL, Hao Y, et al. (2019) Standing genetic variation as the predominant source for adaptation of a songbird. P Natl Acad Sci USA 116: 2152-2157.

22. Ahrens CW, Byrne M, Rymer PD (2019) Standing genomic variation within coding and regulatory regions contributes to the adaptive capacity to climate in a foundation tree species. Molecular Ecology 28: 2502-2516.

23. Rockman MV (2012) The QTN program and the alleles that matter for evolution: all that's gold does not glitter. Evolution 66: 1-17.

24. Chen P, Zhang J (2020) Antagonistic pleiotropy conceals molecular adaptations in changing environments. Nature ecology \& evolution 4: 461-469.

25. Doyle JJ, Doyle JL (1987) A rapid DNA isolation procedure for small quantities of fresh leaf tissue. Phytochemical Bulletin 19(1):11-15.

26. White TJ, Bruns T, Lee S, Taylor J (1990) 38 - AMPLIFICATION AND DIRECT SEQUENCING OF FUNGAL RIBOSOMAL RNA GENES FOR PHYLOGENETICS. In: Innis MA, Gelfand DH, Sninsky JJ, White TJ, editors. PCR Protocols. San Diego: Academic Press. pp. 315-322.

27. Taberlet P, Gielly L, Pautou G, Bouvet J (1991) Universal primers for amplification of three non-coding regions of chloroplast DNA. Plant molecular biology 17: 1105-1109.

28. Oxelman B, Lidén M, Berglund D (1997) Chloroplast rps16 intron phylogeny of the tribe Sileneae (Caryophyllaceae). Plant Systematics and Evolution 206: 393-410.

29. Hurr KA, Lockhart PJ, Heenan PB, Penny D (1999) Evidence for the recent dispersal of Sophora (Leguminosae) around the Southern Oceans: molecular data. Journal of Biogeography 26: 565-577. microsatellite site (STMS) markers in the Musaceae. Mol Ecol 7: 659-663.

31. Crouch HK, Crouch JH, Jarret RL, Cregan PB, Ortiz R (1998) Segregation at microsatellite loci in haploid and diploid gametes of Musa. Crop Sci 38: 211-217. 
32. Martin G, Baurens FC, Droc G, Rouard M, Cenci A, et al. (2016) Improvement of the banana "Musa acuminata" reference sequence using NGS data and semi-automated bioinformatics methods. BMC genomics 17: 243.

33. Schuelke M (2000) An economic method for the fluorescent labeling of PCR fragments. Nat Biotechnol 18: 233-234.

34. Pritchard JK, Stephens M, Donnelly P (2000) Inference of population structure using multilocus genotype data. Genetics 155: 945-959.

35. Falush D, Stephens M, Pritchard JK (2003) Inference of population structure using multilocus genotype data: linked loci and correlated allele frequencies. Genetics 164: 1567-1587.

36. Peakall ROD, Smouse PE (2006) genalex 6: genetic analysis in Excel. Population genetic software for teaching and research. Molecular Ecology Notes 6: 288-295.

37. Peakall R, Smouse PE (2012) GenAlEx 6.5: genetic analysis in Excel. Population genetic software for teaching and research--an update. Bioinformatics 28: 2537-2539.

38. Bean WT, Stafford R, Brashares JS (2012) The effects of small sample size and sample bias on threshold selection and accuracy assessment of species distribution models. Ecography 35: 250-258.

39. Elith* J, H. Graham* C, P. Anderson R, Dudík M, Ferrier S, et al. (2006) Novel methods improve prediction of species' distributions from occurrence data. Ecography 29: 129151.

40. Elith J, Phillips SJ, Hastie T, Dudík M, Chee YE, et al. (2011) A statistical explanation of MaxEnt for ecologists. Diversity and Distributions 17: 43-57. interpolated climate surfaces for global land areas. Int J Climatol 25: 1965-1978.

42. Warren DL, Glor RE, Turelli M (2010) ENMTools: a toolbox for comparative studies of environmental niche models. Ecography 10.1111/j.1600-0587.2009.06142.x.

43. Gent PR, Danabasoglu G, Donner LJ, Holland MM, Hunke EC, et al. (2011) The Community Climate System Model Version 4. J Climate 24: 4973-4991.

44. Watanabe S, Hajima T, Sudo K, Nagashima T, Takemura T, et al. (2011) MIROC-ESM 2010: model description and basic results of CMIP5-20c3m experiments. Geosci Model Dev 4: 845-872.

45. Brown JL, Anderson B (2014) SDMtoolbox: a python-based GIS toolkit for landscape genetic, biogeographic and species distribution model analyses. Methods in Ecology and Evolution 5: 694-700.

46. Schlotterer C, Tobler R, Kofler R, Nolte V (2014) Sequencing pools of individuals mining genome-wide polymorphism data without big funding. Nat Rev Genet 15: 749-763.

577 47. Cox MP, Peterson DA, Biggs PJ (2010) SolexaQA: At-a-glance quality assessment of 
$578 \quad$ Illumina second-generation sequencing data. Bmc Bioinformatics 11.

48. Martin M (2011) Cutadapt removes adapter sequences from high-throughput sequencing reads. EMBnetjournal 17: 10-12.

49. Wu W, Yang YL, He WM, Rouard M, Li WM, et al. (2016) Whole genome sequencing of a banana wild relative Musa itinerans provides insights into lineage-specific diversification of the Musa genus. Sci Rep 6: 31586.

50. Li H, Durbin R (2009) Fast and accurate short read alignment with Burrows-Wheeler transform. Bioinformatics 25: 1754-1760.

51. McKenna A, Hanna M, Banks E, Sivachenko A, Cibulskis K, et al. (2010) The Genome Analysis Toolkit: A MapReduce framework for analyzing next-generation DNA sequencing data. Genome Res 20: 1297-1303.

52. Wu W, Ng WL, Yang JX, Li WM, Ge XJ (2018) High cryptic species diversity is revealed by genome-wide polymorphisms in a wild relative of banana, Musa itinerans, and implications for its conservation in subtropical China. BMC Plant Biol 18: 194. 


\section{Figures:}

\section{$a^{*}$}

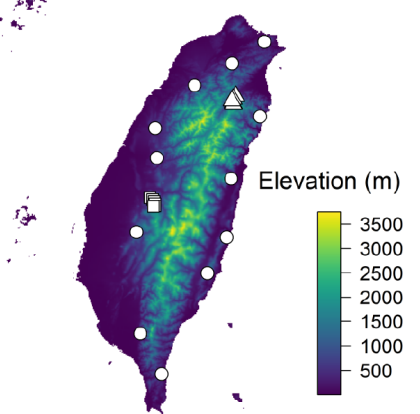

b:

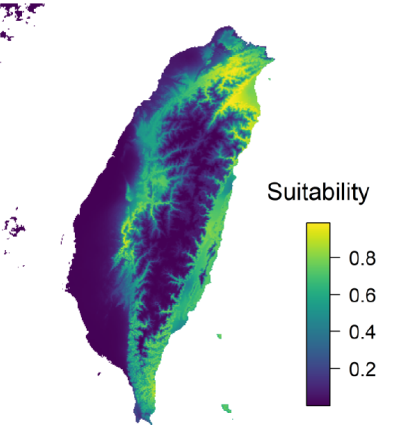

c

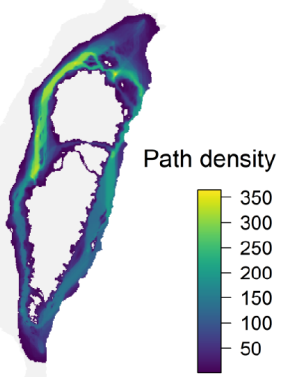

596 Fig. 1 | Sample distribution and niche modeling of Musa itinerans. a, Sampling sites are

597 distributed along the latitudinal and altitudinal gradient. Lowland populations are represented

598 as white circles; transect populations are represented as white triangles and squares. b,

599 Suitability is derived from MaxEnt niche modeling. c, Least-cost-corridor landscape is

600 constructed from pairwise least-cost paths among 20 populations from the resistance surface

601 (the reciprocal of niche suitability). 
a

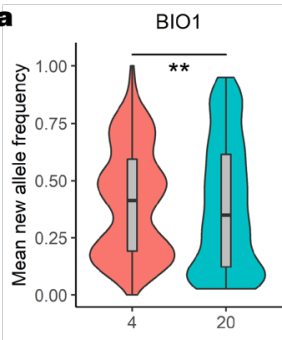

Environment $\square$ Novel $\square$ Ancestral
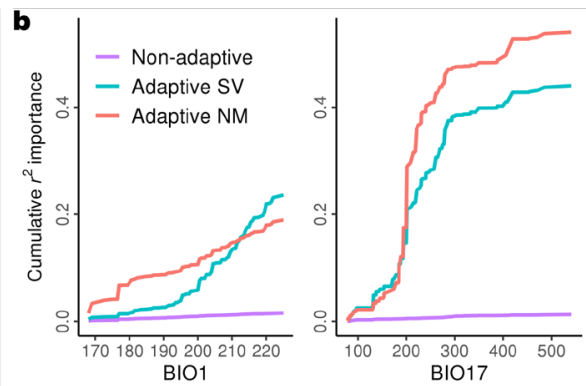

c

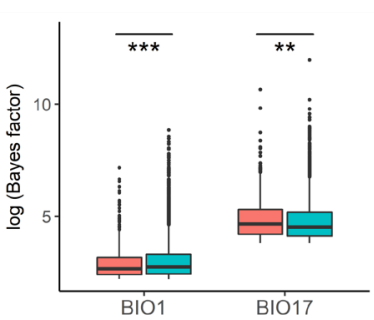

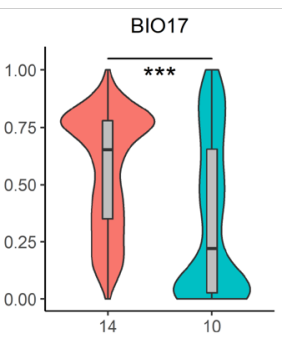

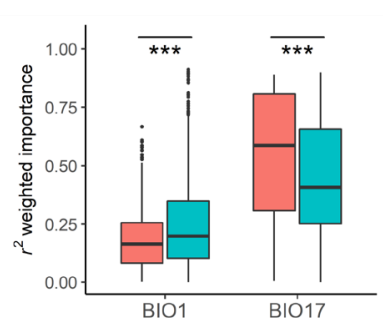

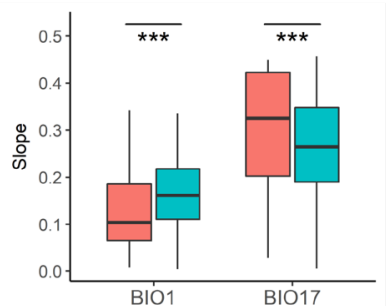

New mutation $\boxminus$ Standing variation

604 Fig. 2 | The environment-dependent enrichment of new alleles and the distinct effect sizes of standing variation (SV) and new mutations (NM) in contrasting climatic factors. BIO1

606 reflects annual mean temperature, and BIO17 indicates precipitation of driest quarter. a, Mean 607 frequency of new alleles among NM SNPs compared between populations that have local 608 environments within or outside of the ancestral climatic range. New alleles are enriched in 609 novel environments $\left(* * P<0.01,{ }^{* * *} P<0.001, t\right.$-test). Values on the horizontal axis denote the 610 number of Taiwanese populations within the ancestral or novel environmental range. $\mathbf{b}$, 611 Gradient forest cumulative $r^{2}$ importance is shown along environmental gradients. c, Effect 612 sizes as estimated from Bayes factor, gradient forest $r^{2}$ importance, and gradient forest slope 613 all show that $\mathrm{SV}$ exhibit higher effect sizes in BIO1 but the reverse in BIO17 (** $P<0.01$, ***P $614<0.001$, Wilcoxon rank-sum test for Bayes factor and $t$-test for $r^{2}$ importance and slope). 

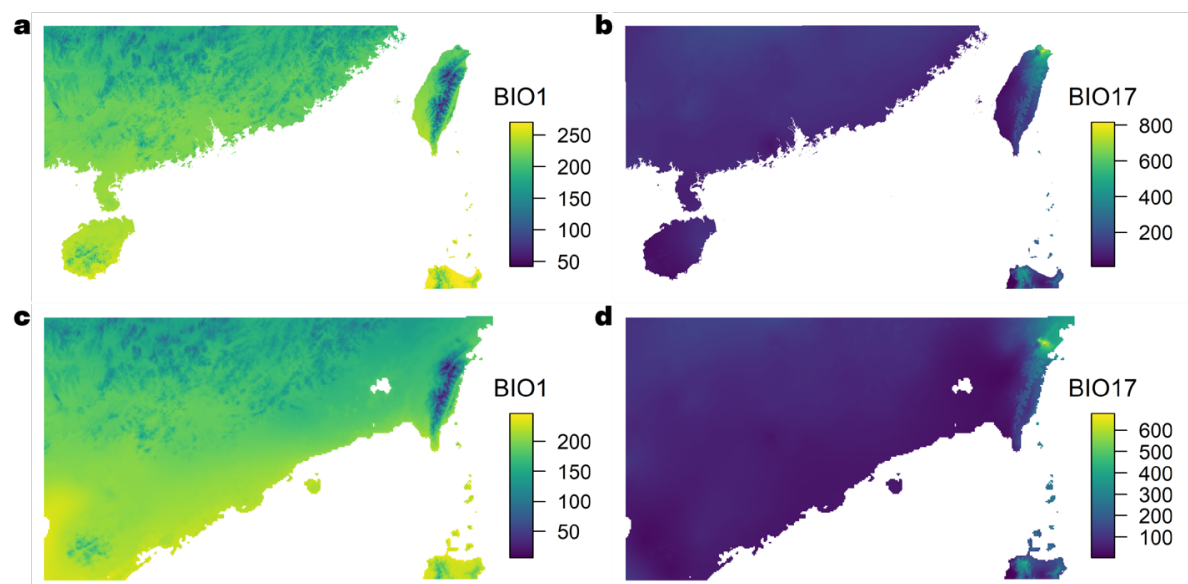

617 Fig. 3 | Annual mean temperature (BIO1) and precipitation of driest quarter (BIO17) for

618 the present and last glacial maximum. a, b, Present environments. c, d, Last-glacial619 maximum environments, showing the environments on the extent of land. Maps on all panels 620 have the same range in latitude and longitude. 

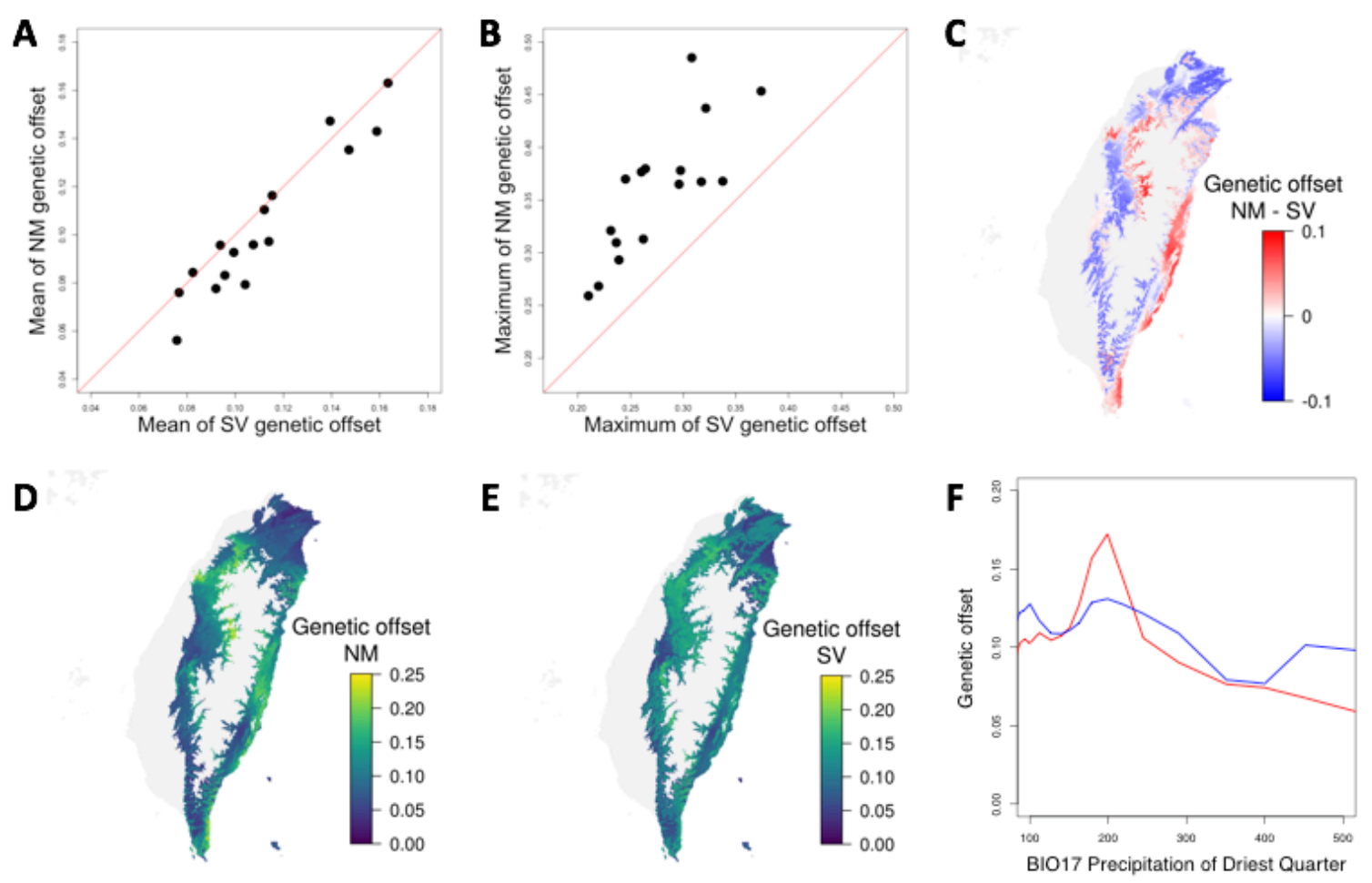

622

623 Fig. 4 | The maladaptation of standing variation (SV) and new mutations (NM) towards

624 anthropogenic climate change. (A) the mean and (B) the maximum genetic offset of currently

625 adaptive SV and NM SNPs across map of Taiwan for each of the 16 future climatic scenarios.

626 In general, SV has higher mean genetic offset and NM has higher maximum genetic offset. (C),

627 for the genetic offset map averaged across all 16 future scenarios, this graph shows the offset

628 from NM minus SV. The values range from -0.07 to 0.1 , representing NM has more extreme

629 genetic offset values than SV as shown in (D) and (E). (D and E), the genetic offset value for

$630 \mathrm{NM}$ and SV, averaged across all 16 future scenarios. (F), genetic offset values for NM (red)

631 and SV (blue) across current gradients of BIO17 (Precipitation of Driest Quarter) across

632 Taiwan. Grids with current niche suitability $<0.2$ are excluded in all calculations and colored

633 in gray in all maps. 
635 Data Availability:

636 Population pooled sequencing reads are available under NCBI BioProject PRJNA575344.

\section{Acknowledgements:}

638 We thank Thomas Mitchell-Olds and Sergey Nuzhdin for valuable comments, Hui-Long Chiu

639 for the knowledge of Musa itinerans ecology in Taiwan, Chia-Yu Chen, Zhe-Ting Kuo, and Jo-

640 Wei Hsieh for the assistance of sample preparation, Hao-Chih Kuo for the introduction to

641 species distribution modeling, Cheng-Tao Lin for providing the R template of some maps, the

642 Lee lab member for valuable discussions, and the Computer and Information Networking

643 Center of National Taiwan University for the support of high-performance computing facilities.

644 This work is supported by the Ministry of Science and Technology of Taiwan (106-2628-B-

645 002-001-MY3, 107-2636-B-002-004, and 108-2636-B-002-004 to CRL).

646 Author Contributions:

647 C-RL designed the research. Both authors performed experiments and analyses and wrote the

648 manuscript.

649 Competing Interest Declaration:

650 None

651 

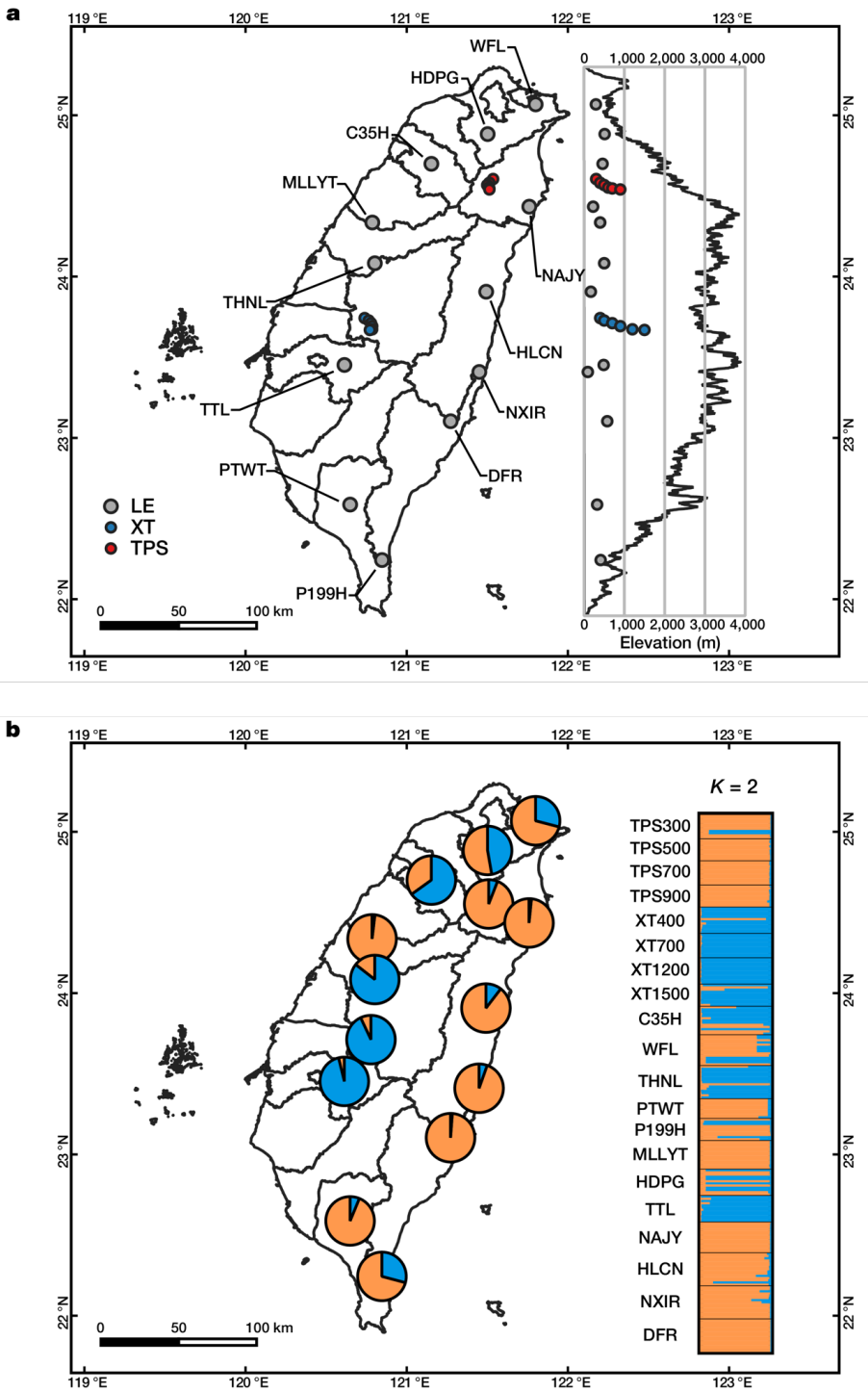

654 Fig. S1 | Sampling site and population structure of Taiwanese Musa itinerans under STRUCTURE $\boldsymbol{K}=2$. a, Solid circles represent collection locations corresponding to their coordinates and elevation: Gray circles indicate populations of low elevation (LE); blue circles indicate populations of Xitou transect (XT); red circles indicate populations of Taipingshan transect (TPS). $\mathbf{b}$, Individual ancestry is plotted on the right side, while population ancestry is plotted on map with a pie chart. Map template is provided by *Cheng-Tao Lin.

$660 *$ Cheng-Tao Lin (2018) QGIS template for displaying species distribution by horizontal and 661 vertical view in Taiwan. URL: https:/github.com/mutolisp/distrmap_tw.qgis. DOI: 
a

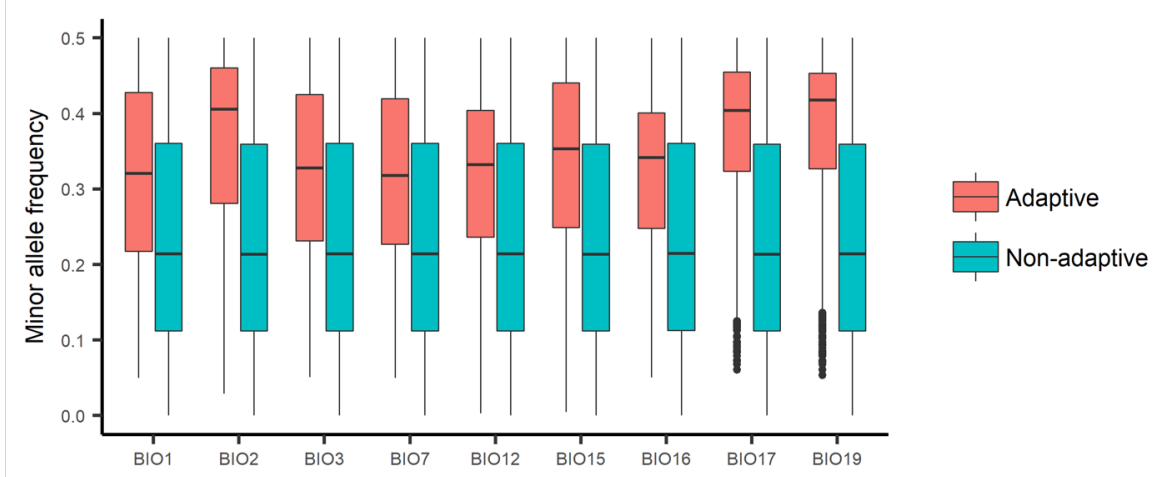

b

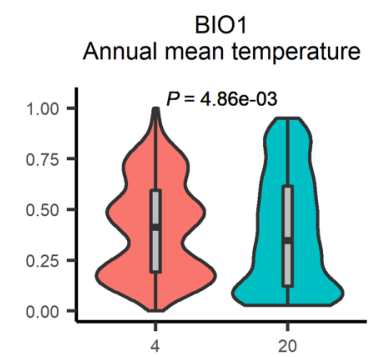

$\mathrm{BIO7}$

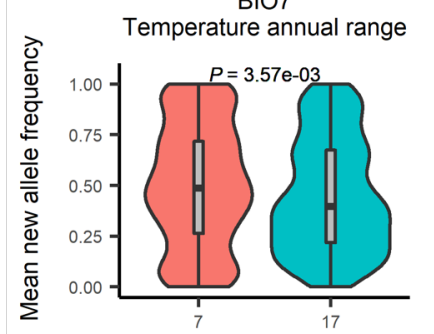

$\mathrm{BIO16}$ Precipitation of wettest quarter

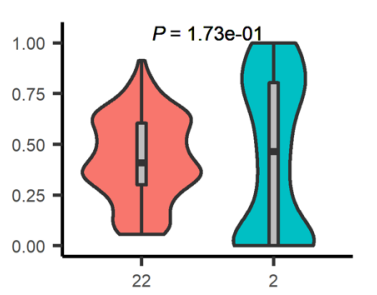

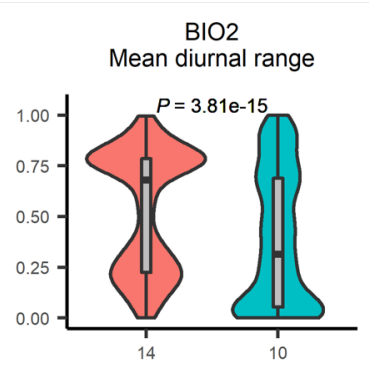

$\mathrm{BIO} 12$ Annual precipitation

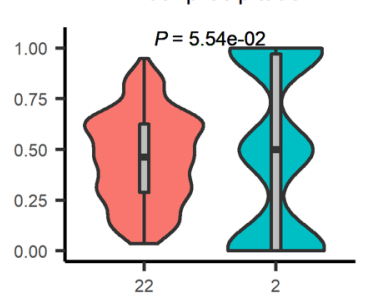

$\mathrm{BIO} 17$

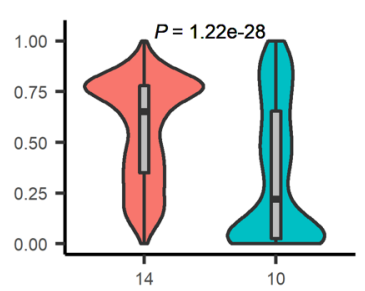
Precipitation of driest quarter

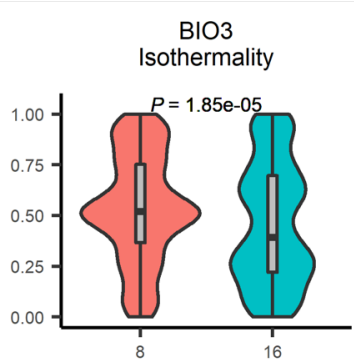

BIO15 Precipitation seasonality

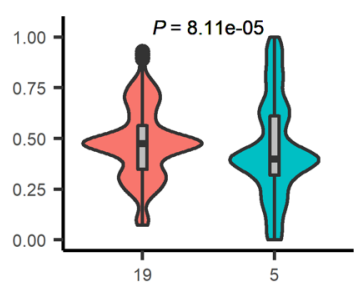

BIO19

Precipitation of coldest quarter

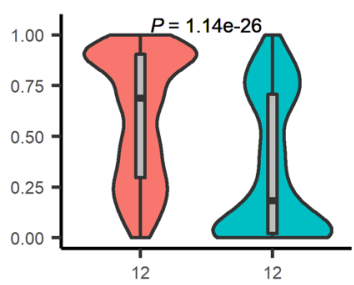

Environment $\square$ Novel $\square$ Ancestral

665 Fig. S2 | Allele frequency distribution. a, Minor allele frequency distribution of adaptive and non-adaptive SNPs. b, Frequency of new alleles in Taiwanese populations within the ancestral or novel environmental range. Statistical significance from Student's $t$-test between the novel and ancestral environmental range for each bioclimatic variable is shown. Values on the

669 horizontal axis denote the number of Taiwanese populations within the ancestral or novel

670 environmental range. 
a

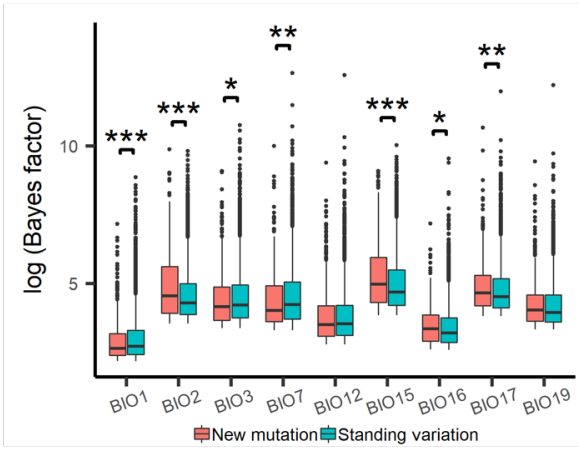

c
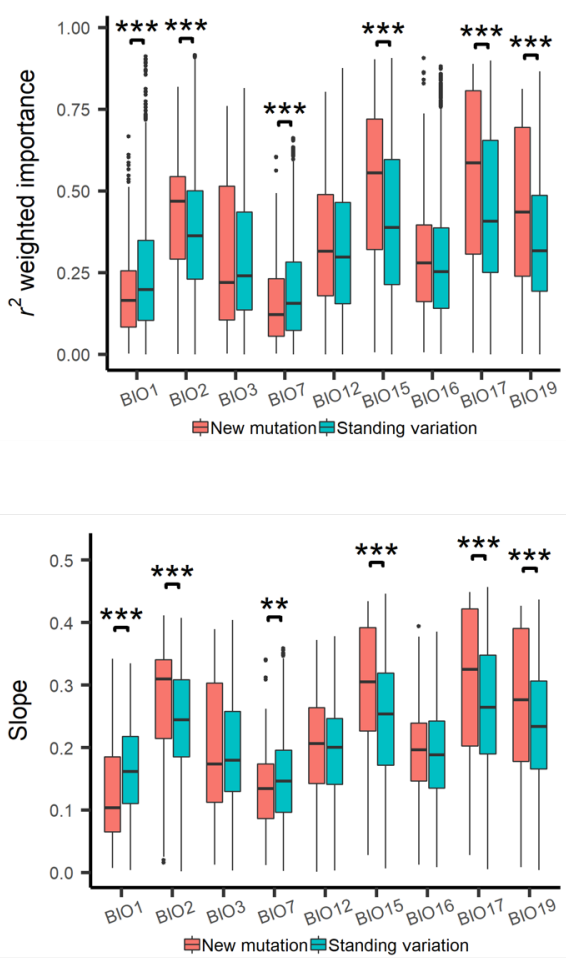

b

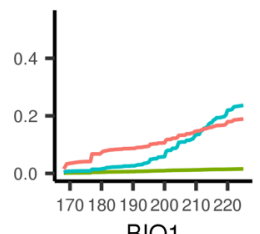

$\mathrm{BIO} 1$

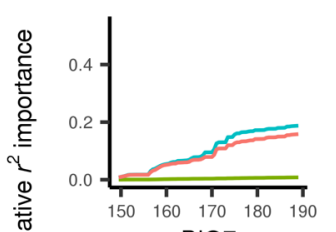

$\mathrm{BIO} 7$

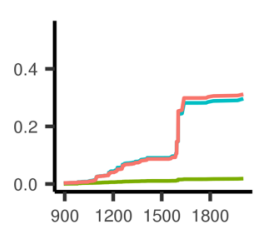

$\mathrm{BIO16}$
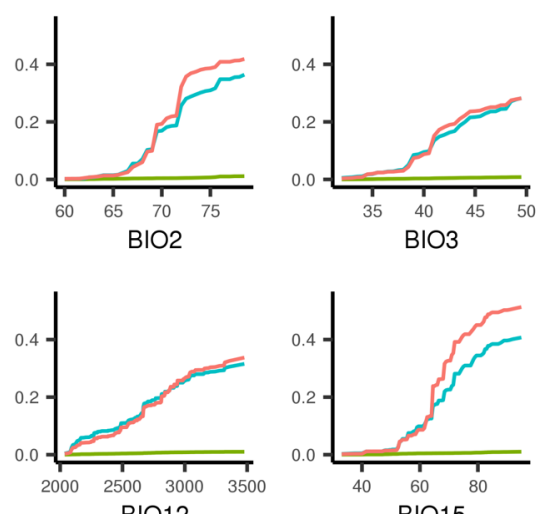

$\mathrm{BIO} 12$

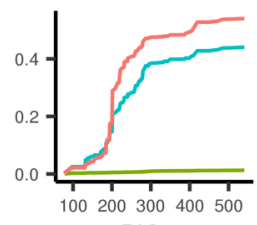

$\mathrm{BIO} 17$

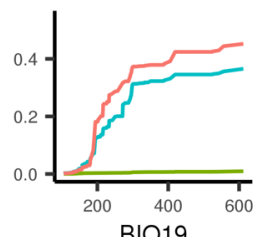

$\longrightarrow$ Non-adaptive $\longrightarrow$ Standing variation (adaptive) $\quad$ New mutation (adaptive)
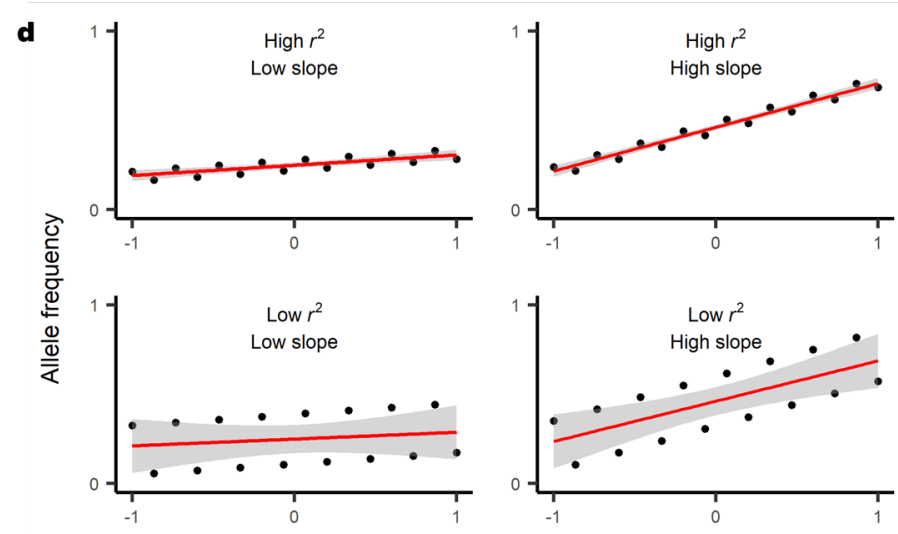

Environmental gradient

672

673 Fig. S3 | Profiling of effect sizes. a, Bayenv Bayes factor distribution for adaptive new

mutations and standing variation $(* P<0.05, * * P<0.01, * * * P<0.001$, Wilcoxon rank-sum test). b, Cumulative $r^{2}$ importance from gradient forest along environmental gradients. c, Gradient forest $r^{2}$ importance distribution (***P<0.001, Welch's $t$-test). d, Example figure showing the relationship between $r^{2}$ importance and slope. $r^{2}$ indicates the extent that allele frequency fits a linear model, while slope indicates the amount of allele frequency changes along the linear relationship. The graphs indicate one should also investigate regression slopes in addition to the gradient forest $r^{2}$. Values on the horizontal axis show the range of standardized environmental variables. e, The distribution of regression slopes when one regresses adaptive SNP allele frequency onto environmental gradients $(* * P<0.01, * * * P<$ 0.001 , Welch's $t$-test). 


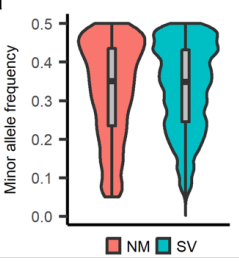

c

\begin{tabular}{cc}
\hline Site & $\begin{array}{c}\text { Expected } \\
\text { heterozygosity }\end{array}$ \\
\hline BX (China) & 0.134 \\
CH (China) & 0.163 \\
HN (China) & 0.140 \\
LC (China) & 0.173 \\
YC (China) & 0.180 \\
C35H & 0.031 \\
DFR & 0.025 \\
HDPG & 0.030 \\
HLCN & 0.039 \\
MLYT & 0.028 \\
NAJY & 0.033 \\
NXII & 0.033 \\
P199H & 0.034 \\
PTWT & 0.025 \\
THNL & 0.036 \\
TPS300 & 0.028 \\
TPS400 & 0.032 \\
TPS600 & 0.034 \\
TPS7700 & 0.033 \\
TPS900 & 0.034 \\
TPS550 & 0.034 \\
TTL & 0.037 \\
WFL & 0.033 \\
XT400 & 0.040 \\
XT500 & 0.036 \\
XT700 & 0.040 \\
XT100 & 0.038 \\
XT1200 & 0.039 \\
XT1500 & 0.043 \\
\hline
\end{tabular}

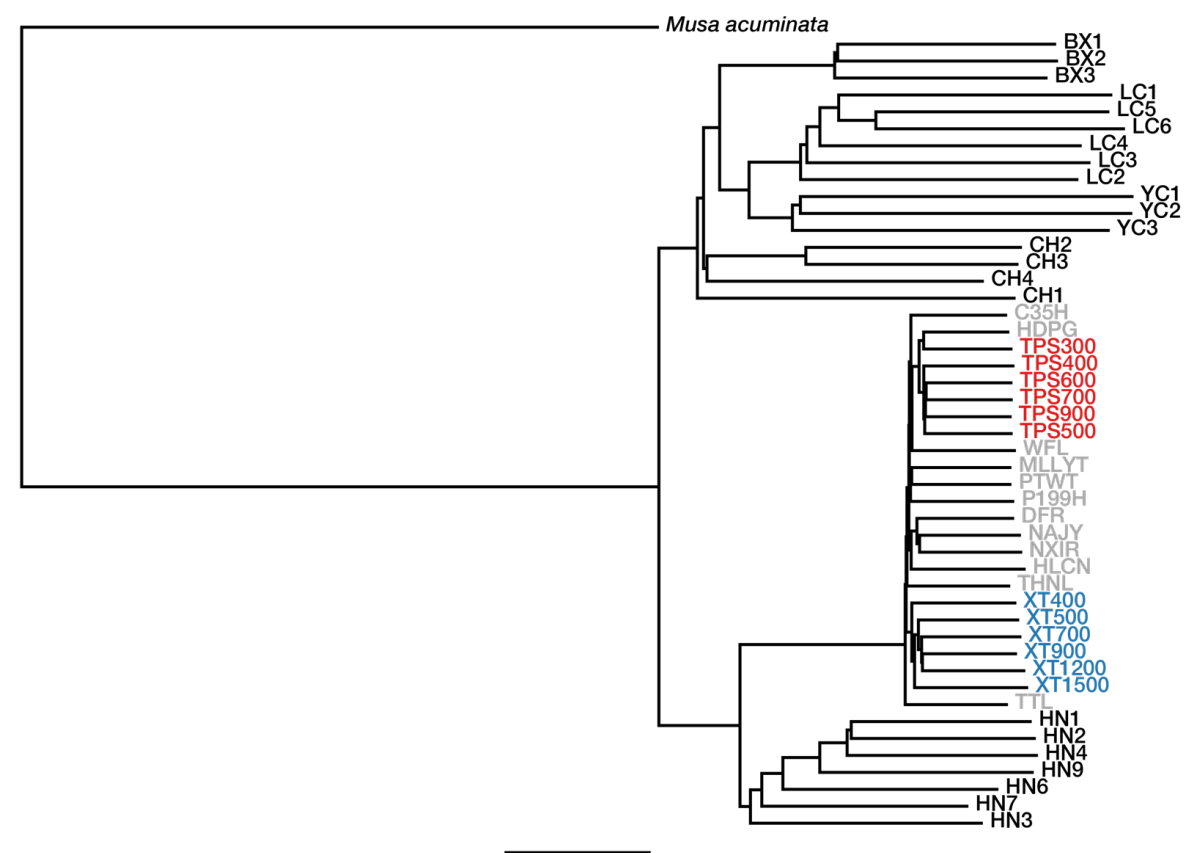

686 Fig. S4 | Evolutionary analyses on Taiwanese and Chinese Musa itinerans. a, Minor allele 687 frequency distribution of adaptive SNPs in Taiwan. $\mathbf{b}$, Phylogeny of Taiwanese and Chinese 688 Musa itinerans. The gray-colored indicates Taiwanese lowland populations (LE); the blue689 colored indicates Taiwanese populations of Xitou transect (XT); the red-colored indicates 690 Taiwanese populations of Taipingshan transect (TPS); the black-colored indicates Chinese 691 accessions and the outgroup Musa acuminata. c, Table showing the expected heterozygosity among Taiwanese and Chinese populations. 
a

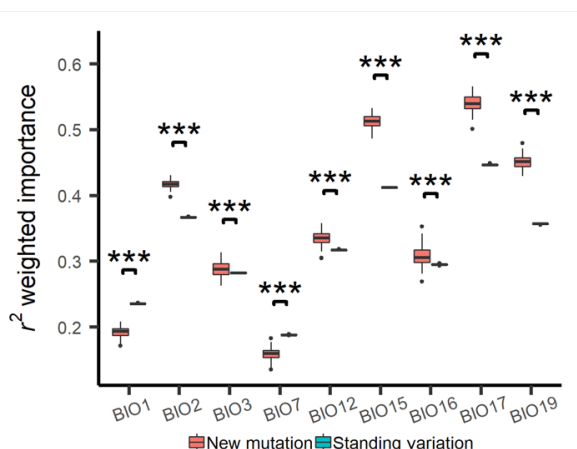

c

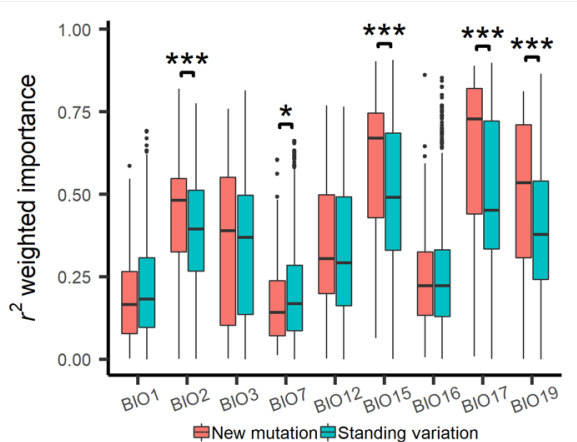

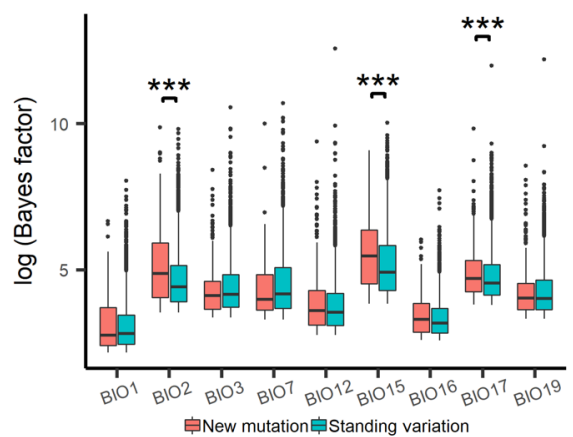

d

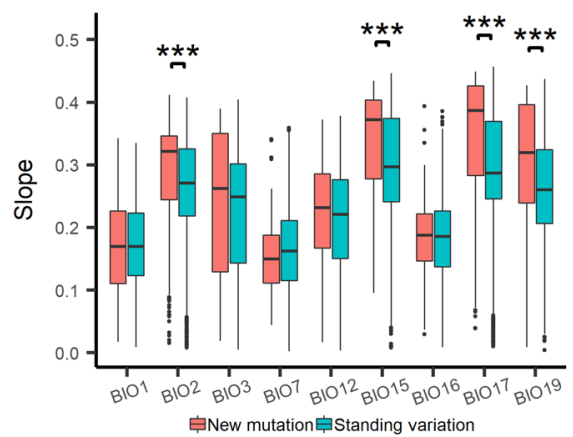

695 Fig. S5 | Multi-analyses addressing ascertainment of standing variation and new mutation.

696 a, The distribution of gradient forest $r^{2}$ importance across 100 re-sampling trials. In each resampling trials, a random set of 50\% new mutations were assigned as standing variation, and

698 the mean $r^{2}$ importance was reported for each trial. All comparisons show strong statistical

699 significance from Student's $t$-test between new mutations and introduced standing variation $700(* * * P<0.001)$. b-d, Profiling of effect sizes of adaptive SNPs with top $50 \%$ minor allele 701 frequency. Distribution of Bayes factor $(* * * P<0.001$, Wilcoxon rank-sum test; b), gradient 702 forest $r^{2}$ importance $\left({ }^{*} P<0.05\right.$, $* * * P<0.001$, Welch's $t$-test; c), and regression slopes of 703 adaptive SNP allele frequency onto environmental gradients $(* * * P<0.001$, Welch's $t$-test; d) is analyzed between new mutations and standing variation for each bioclimatic variable. 

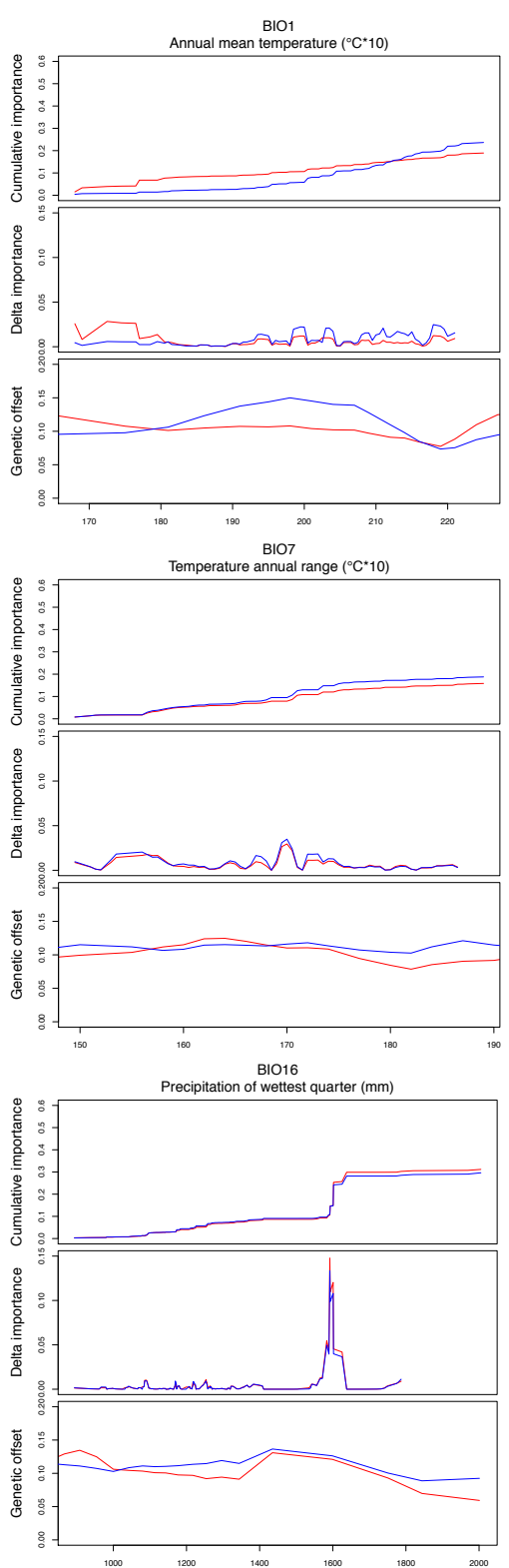
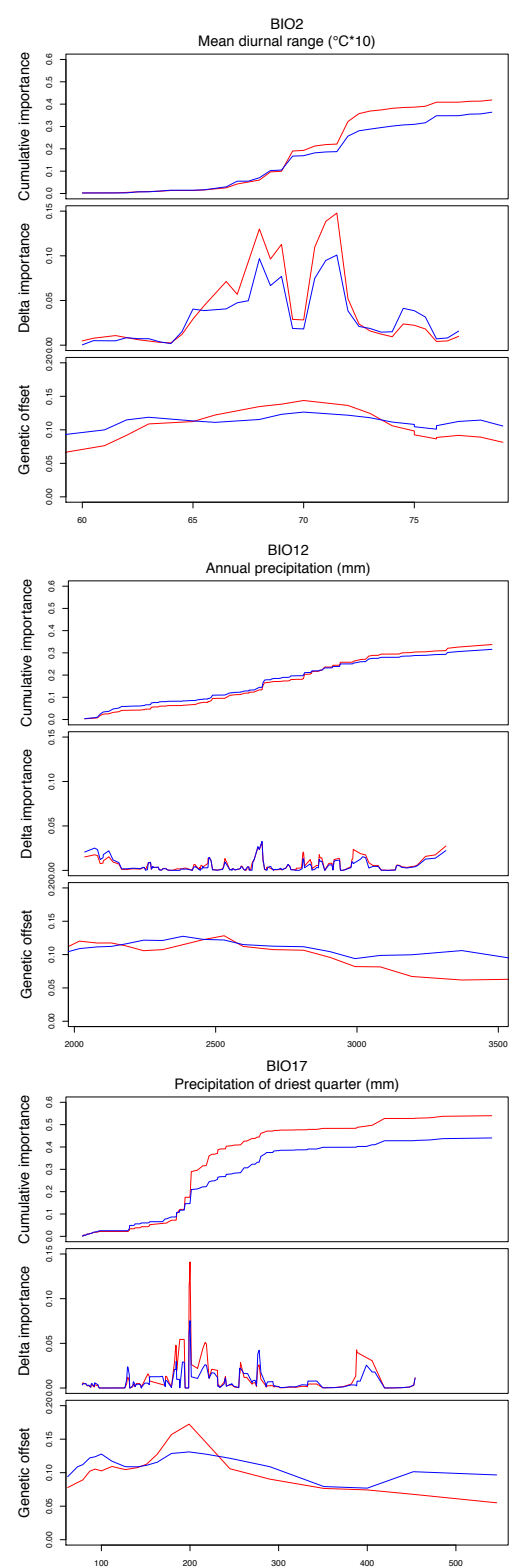
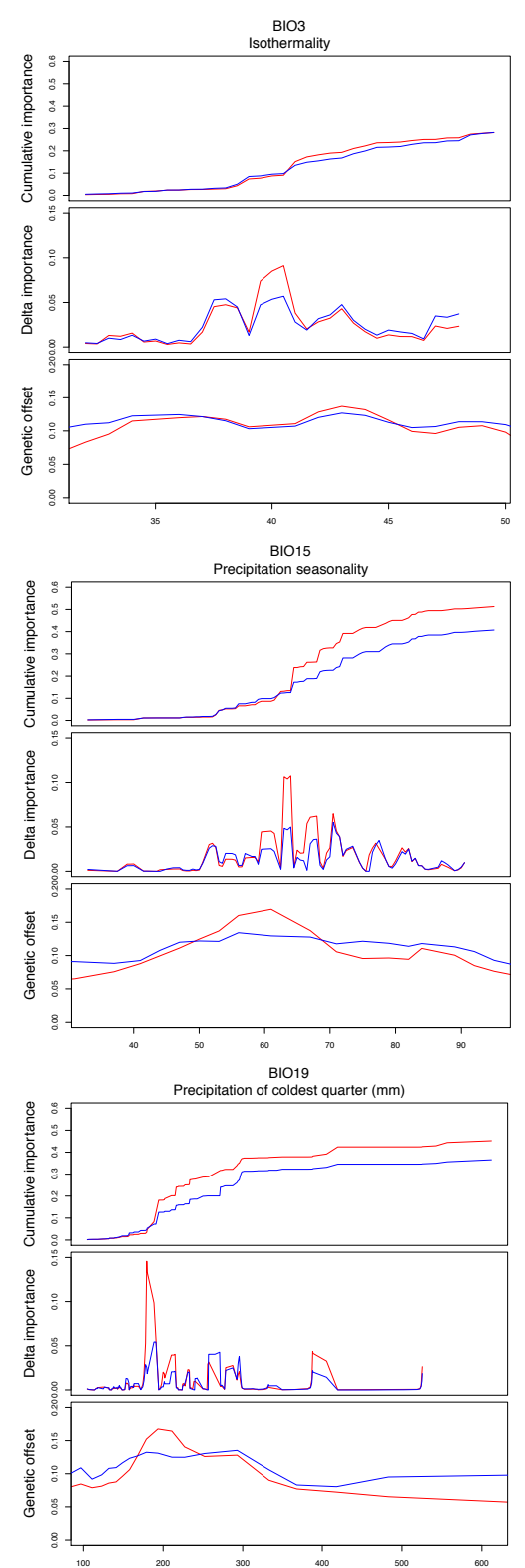

Fig. S6 | Association among gradient forest cumulative importance, delta importance per unit environmental gradient, and genetic offset for each bioclimatic variable. In each figure, red line represents new mutation (NM), and blue line represents standing variation (SV). The "delta importance" is analogous to the instantaneous slope on the cumulative importance graph. Genetic offset was calculated for SV and NM separately, where the genetic offset maps of 16 future climatic scenarios were averaged into one map for SV and one for NM. Geographic grids were separated into 20 bins based on their bioclimatic values, and the mean genetic offset in each bin was plotted. Grids with current niche suitability $<0.2$ are excluded in all calculations and colored in gray in all maps. 
bioRxiv preprint doi: https://doi.org/10.1101/2020 01.12 903427; this version posted March 5, 2020. The copyright holder for this preprint (which was not certified by peer review) is the author/funder, who has granted bioRxiv a license to display the preprint in perpetuity. It is made available under aCC-BY-NC-ND 4.0 International license.

717 Table S1 | Population coordinates and bioclimatic information

\begin{tabular}{cccccccccccc}
\hline Site & Latitude & Longitude & BIO1 & BIO2 & BIO3 & BIO7 & BIO12 & BIO15 & BIO16 & BIO17 & BIO19 \\
\hline TPS300 & 24.60488 & 121.5359 & 208 & 63 & 35 & 180 & 2,970 & 46 & 1,223 & 364 & 364 \\
TPS400 & 24.58135 & 121.5124 & 194 & 65 & 36 & 177 & 2,769 & 50 & 1,145 & 301 & 301 \\
TPS500 & 24.56765 & 121.4992 & 199 & 65 & 36 & 179 & 2,763 & 50 & 1,142 & 299 & 299 \\
TPS600 & 24.55172 & 121.5090 & 193 & 65 & 36 & 176 & 2,709 & 51 & 1,124 & 289 & 289 \\
TPS700 & 24.54638 & 121.5125 & 185 & 65 & 37 & 174 & 2,699 & 49 & 1,107 & 302 & 302 \\
TPS900 & 24.54021 & 121.5126 & 178 & 66 & 38 & 173 & 2,757 & 46 & 1,110 & 336 & 336 \\
XT400 & 23.74292 & 120.7386 & 217 & 77 & 42 & 180 & 2,468 & 86 & 1,395 & 87 & 127 \\
XT500 & 23.72780 & 120.7641 & 206 & 77 & 44 & 173 & 2,600 & 84 & 1,420 & 97 & 141 \\
XT700 & 23.71137 & 120.7782 & 199 & 77 & 45 & 169 & 2,412 & 83 & 1,311 & 94 & 138 \\
XT900 & 23.69294 & 120.7857 & 189 & 77 & 47 & 162 & 2,357 & 83 & 1,287 & 97 & 145 \\
XT1200 & 23.67333 & 120.7854 & 176 & 77 & 48 & 159 & 2,478 & 82 & 1,346 & 97 & 148 \\
XT1500 & 23.66936 & 120.7710 & 160 & 78 & 48 & 160 & 2,637 & 81 & 1,402 & 98 & 149 \\
C35H & 24.69907 & 121.1528 & 202 & 65 & 34 & 188 & 2,500 & 55 & 1,073 & 192 & 289 \\
WFL & 25.06752 & 121.7998 & 203 & 58 & 31 & 185 & 3,440 & 29 & 1,229 & 606 & 749 \\
THNL & 24.08273 & 120.8021 & 212 & 74 & 40 & 184 & 2,188 & 83 & 1,220 & 78 & 138 \\
PTWT & 22.58684 & 120.6489 & 227 & 78 & 49 & 158 & 2,912 & 98 & 1,784 & 79 & 87 \\
P199H & 22.24302 & 120.8465 & 223 & 73 & 50 & 144 & 3,114 & 92 & 1,856 & 178 & 178 \\
MLLYT & 24.33526 & 120.7864 & 211 & 71 & 37 & 190 & 2,056 & 76 & 1,084 & 79 & 148 \\
HDPG & 24.88347 & 121.5019 & 197 & 62 & 33 & 185 & 3,201 & 37 & 1,201 & 475 & 475 \\
TTL & 23.45230 & 120.6134 & 215 & 79 & 45 & 174 & 3,515 & 98 & 2,154 & 81 & 123 \\
NAJY & 24.43352 & 121.7602 & 216 & 62 & 35 & 174 & 2,860 & 61 & 1,328 & 271 & 296 \\
HLCN & 23.90623 & 121.4928 & 213 & 68 & 40 & 168 & 2,127 & 46 & 849 & 251 & 255 \\
NXIR & 23.40887 & 121.4503 & 227 & 71 & 43 & 163 & 2,147 & 57 & 938 & 211 & 211 \\
DFR & 23.10356 & 121.2724 & 206 & 75 & 48 & 155 & 2,017 & 68 & 1,026 & 166 & 166 \\
\hline & & & & & & & & & & &
\end{tabular}


bioRxiv preprint doi: https//doi.org/10.1101/2020.01.12 903427; this version posted March 5,2020 . The copyright holder for this preprint (which was not certified by peer review) is the author/funder, who has granted bioRxiv a license to display the preprint in perpetuity. It is made available under aCC-BY-NC-ND 4.0 International license.

Table S2 | SSR primer information

\begin{tabular}{|c|c|c|c|}
\hline Locus & Primer (5' to $\left.3^{\prime}\right)$ & Chromosome* & Position* \\
\hline CIR436 & $\begin{array}{l}\text { ATAAGCTCATATGGGTACAGTCACA } \\
\text { CTGCAGCAACCAAATTTATTTCT }\end{array}$ & 1 & $\begin{array}{l}4,917,979-4,918,003 \\
4,918,057-4,918,080\end{array}$ \\
\hline CIR276 & $\begin{array}{l}\text { CTCCTCCATAGCCTGACTGC } \\
\text { TGACCCACGAGAAAAGAAGC }\end{array}$ & 1 & $\begin{array}{l}13,588,759-13,588,778 \\
13,588,847-13,588,866\end{array}$ \\
\hline CIR1113 & $\begin{array}{l}\text { ACTCTCGCCCATCTTCATCC } \\
\text { ACTTATTCCCCCGCACTCAA }\end{array}$ & 1 & $\begin{array}{l}28,009,948-28,009,967 \\
28,010,173-28,010,192\end{array}$ \\
\hline Ma3-132 & $\begin{array}{l}\text { TCCCTCTTCAACCAAAGCAC } \\
\text { AACGCGAATGTGTGTTTTCA }\end{array}$ & 2 & $\begin{array}{l}20,365,901-20,365,920 \\
20,366,041-20,366,060\end{array}$ \\
\hline CIR646 & $\begin{array}{l}\text { AACACCGTACAGGGAGTCAC } \\
\text { GATACATAAGGCAGTCACATTG }\end{array}$ & 2 & $\begin{array}{l}23,940,966-23,940,985 \\
23,941,276-23,941,297\end{array}$ \\
\hline Ma-1-17 & $\begin{array}{l}\text { AGGCGGGGAATCGGTAGA } \\
\text { GGCGGGAGACAGATGGAGT }\end{array}$ & 2 & $\begin{array}{l}24,383,391-24,383,408 \\
24,383,488-24,383,506\end{array}$ \\
\hline CIR332a & $\begin{array}{l}\text { ATGACCTGTCGAACATCCTTT } \\
\text { TCCCAACCCCTGCAACCACT }\end{array}$ & 3 & $\begin{array}{l}8,978,575-8,978,595 \\
8,978,831-8,978,848\end{array}$ \\
\hline Ма3-48 & $\begin{array}{l}\text { CCCGTCCCATTTCTCA } \\
\text { TTCGTTGTTCATGGAATCA }\end{array}$ & 5 & $\begin{array}{l}32,672,883-32,672,898 \\
32,673,018-32,673,036\end{array}$ \\
\hline CIR631a & $\begin{array}{l}\text { ATTAGATCACCGAAGAACTC } \\
\text { ATCTTTTCTTATCCTTCTAACG }\end{array}$ & 6 & $\begin{array}{l}33,942,751-33,942,770 \\
33,943,017-33,943,038\end{array}$ \\
\hline CIR16a & $\begin{array}{l}\text { TCATCTCACAATGCTTTCATAGTT } \\
\text { TGGTTGAGTAGATCTTCTTGTGT }\end{array}$ & 8 & $\begin{array}{l}1,234,609-1,234,632 \\
1,234,700-1,234,722\end{array}$ \\
\hline Ma3-103 & $\begin{array}{l}\text { TCGCCTCTCTTTAGCTCTG } \\
\text { TGTTGGAGGATCTGAGATTG }\end{array}$ & 8 & $\begin{array}{l}40,127,782-40,127,800 \\
40,127,910-40,127,929\end{array}$ \\
\hline CIR348b & $\begin{array}{l}\text { ACAGAATCGCTAACCCTAATCCTCA } \\
\text { CCCTTTGCGTGCCCCTAA }\end{array}$ & 10 & $\begin{array}{l}27,228,162-27,228,186 \\
27,228,325-27,228,342\end{array}$ \\
\hline Ma-3-139 & $\begin{array}{l}\text { ACTGCTGCTCTCCACCTCAAC } \\
\text { GTCCCCCAAGAACCATATGATT }\end{array}$ & 10 & $\begin{array}{l}30,237,591-30,237,611 \\
30,237,717-30,237,735\end{array}$ \\
\hline CIR550a & $\begin{array}{l}\text { ACCGCACCTCCACCTCCTG } \\
\text { TGCTGCCTTCATCGCTACTA }\end{array}$ & 10 & $\begin{array}{l}31,914,219-31,914,237 \\
31,914,459-31,914,478\end{array}$ \\
\hline
\end{tabular}

Primer sequences were searched against the Musa acuminata DH-Pahang version $2^{[32]}$ on

723 Banana Genome Hub (https://banana-genome-hub.southgreen.fr/).

724 *The chromosome and position indicate locations on Musa acuminata where primer sequences

725 were found by blast. 
727 Table S3 | Number of new-mutation (NM) or standing-variation (SV) SNPs with (adaptive)

728 or without (non-adaptive) significant associations with bioclimatic variables

\begin{tabular}{ccccccc}
\hline Variable & $\begin{array}{c}\text { Non-adaptive } \\
\text { NM }\end{array}$ & $\begin{array}{c}\text { Non-adaptive } \\
\text { SV }\end{array}$ & $\begin{array}{c}\text { Adaptive } \\
\text { NM }\end{array}$ & $\begin{array}{c}\text { Adaptive } \\
\text { SV }\end{array}$ & $P$ & Odds ratio \\
\hline BIO1 & 293,348 & 954,367 & 816 & 7,113 & $8.92 \mathrm{e}-169$ & 2.68 \\
BIO2 & 293,274 & 953,198 & 890 & 8,282 & $8.43 \mathrm{e}-213$ & 2.86 \\
BIO3 & 293,551 & 953,901 & 613 & 7,579 & $6.47 \mathrm{e}-256$ & 3.80 \\
BIO7 & 293,644 & 953,981 & 520 & 7,499 & $1.32 \mathrm{e}-282$ & 4.44 \\
BIO12 & 293,452 & 954,272 & 712 & 7,208 & $3.12 \mathrm{e}-203$ & 3.11 \\
BIO15 & 293,168 & 951,447 & 996 & 10,033 & $2.33 \mathrm{e}-281$ & 3.10 \\
BIO16 & 293,826 & 957,605 & 338 & 3,875 & $1.95 \mathrm{e}-123$ & 3.52 \\
BI017 & 293,461 & 954,969 & 703 & 6,511 & $1.61 \mathrm{e}-166$ & 2.85 \\
BI019 & 293,516 & 955,326 & 648 & 5,142 & $1.06 \mathrm{e}-107$ & 2.44 \\
\hline
\end{tabular}

Statistical significance from $\chi^{2}$ test is shown for each bioclimatic variable. Odds ratio is

731 calculated as (adaptive SV / adaptive NM) / (non-adaptive SV / non-adaptive NM). 
733 Table S4 | Number of new-mutation (NM) or standing-variation (SV) SNPs with (adaptive) 734 or without (non-adaptive) significant associations with bioclimatic variables (controlled 735 for minor allele frequency)

\begin{tabular}{ccccccc}
\hline Variable & $\begin{array}{c}\text { Non-adaptive } \\
\text { NM }\end{array}$ & $\begin{array}{c}\text { Non-adaptive } \\
\text { SV }\end{array}$ & $\begin{array}{c}\text { Adaptive } \\
\text { NM }\end{array}$ & $\begin{array}{c}\text { Adaptive } \\
\text { SV }\end{array}$ & $P$ & Odds ratio \\
\hline BIO1 & 44,920 & 260,113 & 238 & 2,573 & $1.27 \mathrm{e}-20$ & 1.87 \\
BIO2 & 23,475 & 148,796 & 124 & 1,073 & $1.18 \mathrm{e}-03$ & 1.37 \\
BIO3 & 39,432 & 234,613 & 182 & 2,689 & $2.21 \mathrm{e}-34$ & 2.48 \\
BIO7 & 41,020 & 242,313 & 182 & 2,794 & $4.35 \mathrm{e}-38$ & 2.60 \\
BIO12 & 37,266 & 224,455 & 220 & 2,514 & $3.36 \mathrm{e}-20$ & 1.90 \\
BIO15 & 32,906 & 201,729 & 290 & 2,647 & $1.36 \mathrm{e}-10$ & 1.49 \\
BIO16 & 33,685 & 206,816 & 113 & 1,282 & $2.88 \mathrm{e}-10$ & 1.85 \\
BIO17 & 12,258 & 72,175 & 64 & 614 & $2.26 \mathrm{e}-04$ & 1.60 \\
BIO19 & 11,237 & 66,547 & 41 & 362 & $1.81 \mathrm{e}-02$ & 1.50 \\
\hline
\end{tabular}

737 The number of four sets of SNPs whose minor allele frequency (MAF) ranges from the first

738 quantile of adaptive MAF to the third quantile of non-adaptive MAF separately for each 739 bioclimatic variable is shown. Statistical significance from $\chi^{2}$ test is shown for each 740 bioclimatic variable. Odds ratio is calculated as (adaptive SV / adaptive NM) / (non-adaptive $741 \mathrm{SV} /$ non-adaptive NM). 
743 Table S5 | Number of currently adaptive new-mutation (NM) or standing-variation (SV)

744 SNPs that remain (retention) or lose (disruption) significant associations with 745 environments under all future climate-change scenarios

\begin{tabular}{ccccccc}
\hline Variable & $\begin{array}{c}\text { Disruption } \\
\text { NM }\end{array}$ & $\begin{array}{c}\text { Disruption } \\
\text { SV }\end{array}$ & $\begin{array}{c}\text { Retention } \\
\text { NM }\end{array}$ & $\begin{array}{c}\text { Retention } \\
\text { SV }\end{array}$ & $P$ & Odds ratio \\
\hline BIO1 & 92 & 635 & 127 & 1,176 & $5.11 \mathrm{e}-02$ & 1.30 \\
BIO2 & 34 & 374 & 348 & 2,010 & $6.85 \mathrm{e}-04$ & 0.53 \\
BIO3 & 21 & 269 & 119 & 2,277 & $1.32 \mathrm{e}-01$ & 1.50 \\
BIO7 & 29 & 251 & 128 & 2,393 & $4.53 \mathrm{e}-04$ & 2.20 \\
BIO12 & 65 & 481 & 136 & 1,509 & $1.37 \mathrm{e}-02$ & 1.50 \\
BIO15 & 37 & 365 & 437 & 2,982 & $4.79 \mathrm{e}-02$ & 0.69 \\
BI016 & 26 & 289 & 25 & 237 & $6.93 \mathrm{e}-01$ & 0.85 \\
BI017 & 37 & 354 & 159 & 1,182 & $2.21 \mathrm{e}-01$ & 0.78 \\
BI019 & 28 & 235 & 45 & 480 & $4.14 \mathrm{e}-01$ & 1.30 \\
\hline
\end{tabular}

747 Statistical significance from $\chi^{2}$ test is shown for each bioclimatic variable. Odds ratio is

748 calculated as (retention SV / retention NM) / (disruption SV / disruption NM). 